\title{
An actuarial mathematical model for a new pension philosophy. An application to the accountant pension fund
}

\author{
Anna Attias* \\ Department MEMOTEF \\ Sapienza University of Rome \\ 00161 Rome, Italy \\ and \\ Simona Ciavalini ${ }^{\dagger}$ \\ Enterpreneur \\ and \\ CARla Morrone $\ddagger$ \\ Department of Business and Economics \\ University of Naples Parthenope \\ DISAE University of Naples Parthenope \\ and \\ DANIELA SAITTA $\S$ \\ Department MEMOTEF \\ Sapienza University of Rome \\ 00161 Rome, Italy
}

(Received: Feb 3, 2020)

\begin{abstract}
This paper adapts an actuarial mathematical model, built for the Italian public pension system, based on the law proposal 3035/2009 to the Accountant Pension Fund (CNPADC). The aim is to introduce a new philosophy pension highly correlated with the concept of adequacy for an ambitious social welfare; using the logic of the 3035/2009 proposal, which guarantees a minimum threshold for the replacement rate of the direct pension, this study provides a rigorous actuarial mathematical model that explains a sort of rate of contribution at a tendential equilibrium, in a pay-as-you-go pension system. This model reveals for which parameters it is possible to intervene to maintain the standard of living in retirement.

*e-mail: anna.attias@uniroma1.it

$\dagger$ e-mail: simona.ciavalini@virgilio.it

${ }^{\ddagger}$ e-mail: carla.morrone@uniparthenope.it

$\S$ e-mail: daniela.saitta@uniroma1.it
\end{abstract}


Mathematics Subject Classifications (2015). $91 \mathrm{~B} 15$

Keywords. Demographic equilibrium, sustainability, rate of contribution at a tendential equilibrium, adequacy, two-component pension.

\section{Introduction}

CNPADC is an entity of private law as an association. The aim of the Fund, as established in the article 2 of the Statute, is to perform - with no profit goal - the function of welfare and assistance in favour of Accountant and their relatives, enrolled in the Register 1

Since 2004 the Fund adopts a NDCS (Notional Defined Contribution Scheme) in a pay-as-you-go (PAYG) logic. In addition to the reform of the calculation method, CNPADC has introduced new requirements for the pensions due to age and seniority. In particular, the rate of the subjective and integrative contribution has been increased and the appreciation mechanism of the retirement has been reviewed.

This article refers to data of CNPADC actuarial balance 2017 (data 2016) drew up by Antonio Annibali ${ }^{2}$ with the logic of the Exact Individual Trajectories Method 3

At $31^{\text {st }}$ December 2016, 62.500 accountants (41.667 males and 20.833 females) are enrolled in CNPADC. The retirees, at the same date, are 7.307 (67\% males and $33 \%$ females) of which 3.761 almost active ( $87 \%$ of them are aged 61-80 years).

The registration into the Fund usually happens among 26 and 36 years old and the average age of a member is 46 years old. Even if males are $67 \%$ of the members, in the future we can hypothesize an equality between genders.

The ratio between active members and retirees (the inverse old age dependency ratio) is stable and equal to about 8,7 proving the youth of the Fund; the cluster of members more copious is that of 36-50 years old.

CNPADC - rightly - has the aim of the long-term financial sustainability 4 and adequacy of the performances 5

The operating balance is positive at 50 years, increasing the accumulated assets, which also increases due to the return on own asset.

In 30 years, also due to the demography of the country, a significant increase of retirees will take place and so the ratio between active members and retirees will be close to one in the period 2025-2050 6 , impacting the balance income-

\footnotetext{
${ }^{1}$ Based on current rules, registered accountants who continuously are in practice, providing services as freelance, even if in retirement, must be enrolled in CNPADC.

2 Cfr. [3].

3 Cfr. [1].

4 The article 24, co. 24 of L. 214/2011, with the scope to ensure the balance between income and expenses, provides a 50 year evaluation for actuarial balances.

5 Adequacy is mentioned only once in article 24, L. 214/2011, without giving instructions.

${ }^{6}$ In this time frame, in fact, the baby boomers will be retired ("baby boomers" refers the large number of people born in the period immediately after the World War II until the mid-sixties).
} 
expenses.

In relation to pension adequacy concept the reference is art. 4 of D.M. $29^{\text {th }}$ November 20077 that says in order to verify the adequacy it is necessary to analyse substitution rate with coherent parameters of the demographic, economics and financial technical basis. This kind of analysis must be performed at least every ten years.

The definition of the substitution rate due to CNPADC 8 is "the ratio between the first annual pension and the mean of the last ten reassessed earnings, net of related contributions ${ }^{2}$ and this is the key concept to think about adequacy.

The transition to the NDCS (logically more equitable and conceptually pivotal for the system sustainability) has, as known, reduced the substitution rates for CNPADC retirees that are estimated at full speed as shown in the table 1, extracted from the actuarial balance.

\begin{tabular}{|c|c|}
\hline Age & Gross substitution rate \\
\hline 62 & $20,44 \%$ \\
\hline 63 & $21,69 \%$ \\
\hline 64 & $23,02 \%$ \\
\hline 65 & $24,43 \%$ \\
\hline 66 & $25,95 \%$ \\
\hline 67 & $27,56 \%$ \\
\hline 68 & $29,31 \%$ \\
\hline 69 & $31,18 \%$ \\
\hline 70 & $33,20 \%$ \\
\hline
\end{tabular}

Table 1 - CNPADC gross substitution rates

This situation can partly be improved increasing the subjective contribution rate that chartered accountants have the hypothetical possibility to pay up to $100 \%$ of their revenues. Obviously, this is not the best way and this paper has the aim to actively contribute to think about this issue through a new philosophy that allows to rigorously correlate the PAYG tendential equilibrium rate with its own parameters.

This is in line with "Save Italy" Decree that imposes the equilibrium between income (contributions) and expenses (pensions) without considering assets and returns generated by these latter, as in a PAYG system.

In the first paragraph of this paper, we describe the law proposal; in the second we refer to the theorem of the asymptotic demographic equilibrium and we built the demographic, economic and financial technical bases with which it is possible to shape the medium tendency replacement rate of the Fund, that is the PAYG tendential contribution rate of equilibrium for all pension components; in the third we do an application with actuarial balance data, using MatLab.

\footnotetext{
${ }^{7}$ Determination of the criteria for the preparation of the actuarial balances of the compulsory social security forms.

8 Pension simulation service (PES).

${ }^{9}$ If the number of years from the registration is less, the average is calculated considering the effective period.
} 


\section{The Cazzola-Treu law proposal}

Considering that the method 10 used to calculate pensions has a significant impact on the quantification, the financial unsustainability of the Italian social security system and the lack of adequacy of the future pension performances have encouraged the study of new legislative proposals as the n. 3035 of $11^{\text {th }}$ December 200911. The mentioned bipartisan proposal focuses on the adequacy, with the aim to have a substitution rate able to guarantee, in a perspective of ambitious social welfare ${ }^{12}$, the maintenance of the standard of living at retirement and the sustainability, and it has, as main goal, the homogenisation of the contribution rate of the basic public system (26\% or $28 \% 13$ among all categories of workers.

Article 1 of the proposed law suggests the constitution of a public pension system based on two compulsory pillars that provide for:

- a basic pension financed by general contribution system with the aim to guarantee a minimum service to retirees;

- a pension calculated with the defined contribution method.

The total and gross pension provision provides for a substitution rate on the reference wage of not less than $60 \%$. The social impact of this proposal is significant because of it is the first and the only one that consider the maintenance of standard life in retirement 14 .

The main interest of signatories was to open a debate ${ }^{15}$ considering that a substitution rate equal to $60 \%$ for direct pension means to set a social guarantee clause, also in line with the position of Europe that already in the 2000 Lisbon Counci ${ }^{16}$ in relation to social security, hoped that the States, which are responsible, guarantee "the medium and long-term financial sustainability of pension systems", but also "... the adequacy of pensions ...".

The European Economic and Social Committee specified in the 2009 Report that: "... the post-2010 strategy must be geared towards the social progress, the strengthening and sustainability of social security systems and the fight against poverty ...? $\left[17\right.$ and the same hope is recalled in the Green Paper ${ }^{18}$ Adequacy and

10 Consider that the substitution rate of Italian basic public pension retirees to which the NDCS is the only applied will be between $31 \%$ and $40 \%$; workers who retired with the Defined Benefit scheme, in full compliance with the requirements, have instead a substitution rate greater than $88 \%$; for pro rata retirees, the substitution rate is between $47 \%$ and $72 \%$. Cfr. [16] and [17].

11 Giuliano Cazzola and Tiziano Treu are the two first signatories of the law proposal in the Chamber of Deputies and in the Senate of the Republic of Parliament, respectively.

12 Cfr. [13].

13 Declaration of Tiziano Treu with regard to the opportunity to have a homogeneous contribution rate.

14 The "Save Italy" Decree notes this maintenance just as a principle for the basic public pension.

15 Declaration of Giuliano Cazzola with regard to the proposal applicability.

16 Lisboncouncil.net.

17 Cfr. [10].

18 Cfr. [11]. 
sustainability are the concepts that European Commission strongly recommends to OECD countries still now ${ }^{19}$ speaking about pension systems.

\section{The actuarial mathematical model of the law proposal adjusted to CNPADC}

The building of the actuarial mathematical model for CNPADC, based on the ones built in $2012^{20}$ and in $2012^{21}$ focuses on the adequacy proposed by CazzolaTreu and takes into account:

- a single contribution rate for the compulsory security system;

- the general assimilation of the calculation rate to the contribution rate;

- a compulsory pension scheme divided into two components;

- the possession of 10 years of contribution;

- the possession of personal requirement data.

In order to build the model, in addition to the standard of CNPADC, it has been considered:

- the asymptotic demographic equilibrium theorem that allows to identify the existence of an asymptotic distribution of the population per age group ${ }^{22}$,

- the flexible substitution rate for contribution and retirement ag $\AA^{23}$

- the actuarial mathematical model for the Cazzola-Treu law proposal for the public pension system 24 .

- the actuarial mathematical model comparing the Cazzola-Treu and the Fornero system: ${ }^{25}$.

- at least 10 years of contribution even not seamless (in antithesis with the 20 years provided by Fornero);

- a substitution rate for the direct pension of at least $60 \%$.

\footnotetext{
19 Cfr. [12].

${ }^{20}$ Cfr. [5].

${ }^{21}$ Cfr. [8].

${ }^{22}$ Cfr. [2].

23 Cfr. [4].

${ }^{24}$ Cfr. [5]

25 Cfr. [6].
} 


\subsection{Recall to demographic asymptotic equilibrium theo- rem}

The asymptotic demographic equilibrium theorem, whose demonstration uses the spectral theory of non-negative matrices ${ }^{26}$ and allows to identify the existence of an asymptotic population distribution by age grour ${ }^{27}$ is at the base of a PAYG DC pension system model as for CNPADC.

Starting from the analysis of classic Leslie mode 28 that does not distinguish between females and males, the variables to be considered are the population divided into $\Omega$ groups of age (assessed in years), with $\Omega \in \mathrm{N}, x_{i}(t)$ the number of individuals of age in $\left[i, i+1\right.$ ), with $i=0,1, \ldots, \Omega-1$ (years), $\alpha_{i} \geq 0$ the birth rate of an individual belonging to group $i, \omega_{i}$ the survival probability of an individual of age $i$, to age $i+1, x(t)=\left[x_{0}(t), x_{1}(t), \ldots, x_{\Omega-1}(t)\right]^{T}$ the population state vector.

Therefore, we can build the Leslie matrix and the following dynamics for the population:

$$
L=\left[\begin{array}{ccccc}
\alpha_{0} & \alpha_{1} & \ldots & \alpha_{\Omega-2} & \alpha_{\Omega-1} \\
\omega_{0} & 0 & \ldots & 0 & 0 \\
0 & \omega_{1} & & 0 & 0 \\
. & \cdot & \ldots & . & \cdot \\
0 & 0 & & \omega_{\Omega-2} & 0
\end{array}\right], x(t+1)=L x(t) \text { with } t=0,1,2, \ldots
$$

Supposing that each person of age $j$ is able to enrol $\alpha_{i} \geq 0$ new members, that is $x(t+1)=\sum_{j=26}^{36} \alpha_{j} \cdot x_{j}(t)$, the number of new members is proportional to inscribed members. It is supposed that $\alpha$ is the same for each age class in relation to the fact that it is absolutely indifferent for convergency.

Data used for the model are, as mentioned, those of the actuarial balance of CNPADC available. In particular:

- maximum achievable age is 105 (against 116 years-old of all Italian population) so $i=26, \ldots, 104$;

- $x^{F}(t)$ and $x^{M}(t)$ are population state vectors for females and males, respectively;

- $q_{i}^{F}$ and $q_{i}^{M}$ are mortality rates for females and males of age $i$;

- $\alpha_{i}^{F}$ and $\alpha_{i}^{M}{ }^{29}$ are registration rates to CNPADC by gender ${ }^{30}$

- $\omega_{i}^{F}=1-q_{i}^{F}$ and $\omega_{i}^{M}=1-q_{i}^{M}$ are the survival probability for females and males.

\footnotetext{
${ }^{26}$ Cfr. [7].

27 Cfr. [2].

${ }^{28}$ Cfr. [14] and [15]

29 Adapting the model to CNPADC we do not consider whole population's birth and fertility rates but rather we refer to the specific population of CNPADC whose entrances are usually between 26 and 36 years of age.

30 We assume a registration rate equal to 0 for groups of age outside this range.
} 
Leslie matrix females and males (80x80) for CNPADC and the respective evolution equations are built as follows:

$$
\begin{aligned}
& L^{F}=\left[\begin{array}{ccccccc}
\alpha_{26}^{F} & \ldots & \ldots & \alpha_{36}^{F} & \ldots & \ldots & 0 \\
\omega_{26}^{F} & \ldots & \ldots & 0 & \ldots & \ldots & . \\
. & \ldots & \ldots & . & \ldots & \ldots & . \\
0 & \ldots & \ldots & \omega_{36}^{F} & \ldots & . & 0 \\
. & \ldots & \ldots & . & \ldots & . & . \\
0 & \ldots & \ldots & \ldots & \ldots & \omega_{103}^{F} & 0
\end{array}\right] \\
& x^{F}(t+1)=L^{F} x^{F}(t) \quad t=1, \ldots, 5 q^{31} \\
& L^{M}=\left[\begin{array}{ccccccc}
\alpha_{26}^{M} & \ldots & \ldots & \alpha_{36}^{M} & \ldots & \ldots & 0 \\
\omega_{26}^{M} & \ldots & \ldots & 0 & \ldots & \ldots & . \\
. & \ldots & \ldots & . & \ldots & \ldots & . \\
0 & \ldots & \ldots & \omega_{36}^{M} & \ldots & . & 0 \\
. & \ldots & \ldots & . & \ldots & . & . \\
0 & \ldots & \ldots & \ldots & \ldots & \omega_{103}^{M} & 0
\end{array}\right] \\
& x^{M}(t+1)=L^{M} x^{M}(t) \quad t=1, \ldots, 50 .
\end{aligned}
$$

We can now determine the total population state vector $x=\left[\begin{array}{c}x^{F} \\ x^{M}\end{array}\right]$ and the resulting dynamics $x(t+1)=\Lambda x(t)$, where $\Lambda=\left[\begin{array}{cc}L^{F} & 0 \\ d & 0 \\ 0 & L_{1}^{M}\end{array}\right]$ is the $160 \times 160$ block matrix, in which

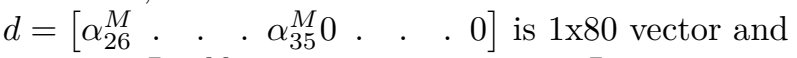

$$
\begin{aligned}
& L_{1}^{M}=\left[\begin{array}{cccccc}
\omega_{26}^{M} & \ldots & \ldots & \ldots & 0 & 0 \\
\cdot & \ldots & \ldots & \ldots & . & . \\
. & \ldots & \ldots & \ldots & . & . \\
0 & \ldots & \ldots & \ldots & \omega_{103}^{M} & 0
\end{array}\right] \text { is } 79 \times 80 \text { matrix. }
\end{aligned}
$$

Although the system matrix of the two-sex model does not satisfy the conditions of the classical Perron-Frobenius theorem, it can be proved that the age distribution of the population converges to a demographic equilibrium, for the sub-population of females $0-50$ years, and the result can be extended to the whole population, hence we obtain the following theorem 3

Theorem 1. For the whole population, there exist a demographic equilibrium, that is a population state $x^{0}$ which is a non-negative eigenvector of matrix $\Lambda$, associated with a unique positive eigenvalue $\lambda_{0}$. On the long run, the age

\footnotetext{
${ }^{31}$ In the actuarial balance the forecast is done for 50 years, so this is the timeframe to measure the effects of the Cazzola-Treu law proposal.

32 Cfr. [2].
} 
AN ACTUARIAL MATHEMATICAL MODEL FOR A NEW PENSION PHILOSOPHY... 33

distribution of the population tends to the equilibrium age distribution in the sense that for any $x(0)$ there exists a number $s>0$ such that $\lim _{t \rightarrow \infty} \frac{x(t)}{\lambda_{0}^{t}}=s x^{0}$.

Hypothesizing a demographic equilibrium, we want to estimate the average trend substitution rate for all pension components, obtained with the sum of the two portions of pension. Intending to impose that, at full speed, the ratio between average direct pensions and average earnings is at least 0,6 , it needs to operate on all pension structure and assess, to set up which are actual ranges of variables variations contribute to pension formation. The contribution rate, as mentioned, is set a priori in the logic of the law but, once the model has been constructed, other hypotheses can be tested with respect to the rate measure itself to obtain the purpose of the adequacy of the pension, that is the fact that the relationship built with the direct pensions must generate a substitution rate of $60 \%$.

\subsection{Demographic technical bases}

\subsubsection{Active and retired population dynamics}

The population of CNPADC can be discerned in active and retired by gender where $l_{i}^{F}(h, t)$ and $l_{i}^{M}(h, t)$ are respectively the number of active women and men of age $i$, at time $t$, with $h(1 \leq h \leq H)$ years of contribution to CNPADC, $H$ is the maximum period of contribution, that is 50 years, with $l_{i}^{F}(h, t)=$ $l_{i}^{M}(h, t)=0$, if $\left\{\begin{array}{l}0 \leq i \leq h+26 \\ 70 \leq i \leq 104\end{array}\right.$.

In a static hypothesis, distinguishing by gender, we have:

- ${ }_{\text {dir }}^{\text {new }} P_{i}^{F}(h, t)$ and ${ }_{\text {dir }}^{\text {new }} P_{i}^{M}(h, t)$ new direct retirees;

- ${ }_{\text {dis }}^{\text {new }} P_{i}^{F}(h, t)$ and ${ }_{\text {dis }}^{\text {new }} P_{i}^{M}(h, t)$ new retirees with disability;

- ${ }_{\text {ina }}^{\text {new }} P_{i}^{F}(h, t)$ and ${ }_{\text {ina }}^{\text {new }} P_{i}^{M}(h, t)$ new retirees with inability.

The minimum contributory seniority - as provided for by Cazzola-Treu - is 10 years and it is combined with minimum requirements of CNPADC.

The balance equation for active females is:

$l_{i+1}^{F}(h+1, t+1)=l_{i}^{F}(h, t) \omega_{i}^{F}-{ }_{\operatorname{dir}}^{n e w} P_{i}^{F}(h, t)-{ }_{\text {dis }}^{\text {new }} P_{i}^{F}(h, t)-{ }_{\text {ina }}^{n e w} P_{i}^{F}(h, t)$. Indicating with $\tau_{i}^{F}(t)$ the first registration rate to CNPADC and with $\tau^{F}(t)=$ $\left(\tau_{0}^{F}(t), \tau_{1}^{F}(t), \ldots, \tau_{104}^{F}(t)\right)$ the related vector, ${ }^{n e w} l^{F}(t)=x^{F}(t) \circ \tau^{F}(t){ }^{33}$ is the female new members vector.

If we suppose ${ }^{\text {new }} P_{i}^{F}(h, t)={ }_{\text {dir }}^{\text {new }} P_{i}^{F}(h, t)+{ }_{\text {dis }}^{\text {new }} P_{i}^{F}(h, t)+{ }_{\text {ina }}^{\text {new }} P_{i}^{F}(h, t)$, we

- for $h=1 l_{i+1}^{F}(1, t+1)={ }^{n e w} l_{i}^{F}(t) \omega_{i}^{F}$;

- for $h>1 l_{i+1}^{F}(h+1, t+1)=l_{i}^{F}(h, t) \omega_{i}^{F}-{ }^{n e w} P_{i}^{F}(h, t)$;

- the retirement rate is $\gamma_{i}(h, t)$.

The formalization is similar for the male population.

$33 \circ$ indicates the Hadamard product. 


\subsubsection{Early retirement and old-age pensioners}

In order to determine the number of new retirees, it needs to consider that two following conditions must be verified to receive the early retirement ${ }^{34}$ and old age pension ${ }^{35}$. $h \geq 25 ; 61 \leq i \leq d^{36}$

Considering direct retirees females and males ${ }_{\text {dir }}^{\text {new }} P_{i}^{F}(h, t)=\gamma_{i}(h, t) l_{i}^{F}(h, t)$, ${ }_{\operatorname{dir}}^{\text {new }} P_{i}^{M}(h, t)=\gamma_{i}(h, t) l_{i}^{M}(h, t)$, the total of new direct retirees is ${ }_{\text {dir }}^{\text {new }} P_{i}(h, t)=$ ${ }_{\text {dir }}^{\text {new }} P_{i}^{F}(h, t)+{ }_{\text {dir }}^{\text {new }} P_{i}^{M}(h, t)$.

Adding up contribution age, the number of new direct retirees of age $i$ is ${ }_{\operatorname{dir}}^{\text {new }} P_{i}^{F}(t)=\sum_{h=1}^{H} \operatorname{dir}_{\operatorname{dir}} P_{i}^{F}(h, t)$ and ${ }_{\operatorname{dir}}^{\text {new }} P_{i}^{M}(t)=\sum_{h=1}^{H} \operatorname{dir}_{i}^{\text {new }} P_{i}^{M}(h, t)$, so ${ }_{\operatorname{dir}}^{\text {new }} P_{i}(t)=$ ${ }_{\text {dir }}^{\text {new }} P_{i}^{F}(t)+{ }_{\operatorname{dir}}^{\text {new }} P_{i}^{M}(t)$.

The recurring dynamics for old direct retirees of age $i$ in year $t$ is

${ }_{\text {dir }}^{\text {old }} P_{i+1}^{F}(t+1)=\left({ }_{\operatorname{dir}}^{\text {old }} P_{i}^{F}(t)+{ }_{\text {dir }}^{\text {new }} P_{i}^{F}(t)\right) \omega_{i}^{F}$ and

${ }_{\text {dir }}^{\text {old }} P_{i+1}^{M}(t+1)=\left({ }_{\text {dir }}^{\text {old }} P_{i}^{M}(t)+{ }_{\text {dir }}^{\text {new }} P_{i}^{M}(t)\right) \omega_{i}^{M}$.

The total of old direct retirees is ${ }_{d i r}^{\text {old }} P_{i}(t)={ }_{d i r}^{\text {old }} P_{i}^{F}(t)+{ }_{d i r}^{\text {old }} P_{i}^{M}(t)$.

The total of direct retirees of age $i$ in year $t$ is ${ }_{\text {dir }} P_{i}(t)={ }_{\text {dir }}^{\text {new }} P_{i}(t)+{ }_{\text {dir }}^{\text {old }} P_{i}(t)$.

Please consider that although CNPADC provides for new members as at $1^{\text {st }}$ January 2004 a further benefit if $i \geq 61$ and $h \geq 5$, the actuarial mathematical model does not consider it.

\subsubsection{Disability and inability pensioners}

Adapting the original model to CNPADC, we considered the difference between inability and disability pensions. Disability retirees may be discerned those one due to disease and injury. Denoting by ${ }^{d i s-d i s e} \psi_{i}^{F}$ and ${ }^{d i s-d i s e} \psi_{i}^{M}$ the probability of an active female and male to be disabled due to disease, with $10 \leq h<a-26$ the new disabled (by disease) female and male retirees are ${ }_{\text {dis-dise }}^{n e w} P_{i+1}^{F}(h, t+1)=l_{i}^{F}(h, t) \omega_{i}^{F \text { dis-dise }} \psi_{i}^{F}$ and ${ }_{\text {dis-dise }}^{\text {new }} P_{i+1}^{M}(h, t+1)=$ $l_{i}^{M}(h, t) \omega_{i}^{M \text { dis-dise }} \psi_{i}^{M}$.

Indicating with ${ }^{d i s-i n j} \psi_{i}^{F}$ and ${ }^{d i s-i n j} \psi_{i}^{M}$ the probability of an active female and male to be disabled due to injury, with $5 \leq h<a-26$, the new disabled (for injury) female and male retirees are

$$
{ }_{\text {dis-inj }}^{\text {new }} P_{i+1}^{F}(h, t+1)=l_{i}^{F}(h, t) \omega_{i}^{F} \text { dis-inj } \psi_{i}^{F}
$$

and ${ }_{\text {dis-inj }}^{n e w} P_{i+1}^{M}(h, t+1)=l_{i}^{M}(h, t) \omega_{i}^{M d i s-i n j} \psi_{i}^{M}$.

The corresponding total of females and males, adding up with respect to seniority, is for disease and injury, respectively:

- ${ }_{\text {dis-dise }}^{\text {new }} P_{i}^{F}(t)=\sum_{h=1}^{H}$ new dis-dise $P_{i}^{F}(h, t)$;

$$
{ }_{\text {dis-dise }}^{n e w} P_{i}^{M}(t)=\sum_{h=1}^{H} \text { dis-dise } P_{i}^{M}(h, t) ;
$$

34 To obtain the early retirement pension in the CNPADC, the minimum contribution requirement is 38 years and the age requirement is 61 years (which is an anomalous thing considering you should therefore enter at age 23). With 40 years of contribution you are entitled to the pension regardless of age.

35 To obtain the old-age pension in CNPADC, you have to be 70 years old and have at least 25 years of contribution.

${ }^{36}$ Even though in CNPADC the old retirement is provided for at 70 years old, members can decide to be active till 105 years old. Mostly active retirees are between 70 and 80 years old. 
- ${ }_{\text {dis-inj }}^{\text {new }} P_{i}^{F}(t)=\sum_{h=1}^{H} \underset{\text { dis-inj }}{\text { new }} P_{i}^{F}(h, t)$;

${ }_{\text {dis-inj }}^{\text {new }} P_{i}^{M}(t)=\sum_{h=1}^{H} \underset{\text { dis-inj }}{\text { new }} P_{i}^{M}(h, t)$.

The sum by gender at age $i$ in year $t$ is respectively

${ }_{\text {dis }}^{\text {new }} P_{i}^{F}(t)={ }_{\text {dis-dise }}^{\text {new }} P_{i}^{F}(t)+{ }_{\text {dis-inj }}^{\text {new }} P_{i}^{F}(t),{ }_{\text {dis }}^{\text {new }} P_{i}^{M}(t)={ }_{\text {dis-dise }}^{\text {new }} P_{i}^{M}(t)+{ }_{\text {dis-inj }}^{\text {new }} P_{i}^{M}(t)$. Adding up by gender ${ }_{\text {dis }}^{\text {new }} P_{i}(t)={ }_{\text {dis }}^{\text {new }} P_{i}^{F}(t)+{ }_{\text {dis }}^{\text {new }} P_{i}^{M}(t)$.

The surviving probability of a disable in the next year is ${ }^{d i s} \bar{\omega}_{i}^{F}$ for females and ${ }^{d i s} \bar{\omega}_{i}^{M}$ for males. Therefore,

$$
\begin{aligned}
& \left.{ }_{\text {dis }}^{\text {old }} P_{i+1}^{F}(h, t+1)={ }_{\text {dis }}^{\text {old }} P_{i}^{F}(h, t)+{ }_{\text {dis }}^{\text {new }} P_{i}^{F}(h, t)\right){ }^{d i s} \bar{\omega}_{i}^{F} \text { and } \\
& \quad{ }_{\text {dis }}^{\text {old }} P_{i+1}^{M}(h, t+1)=\left({ }_{\text {dis }}^{\text {old }} P_{i}^{M}(h, t)+{ }_{\text {dis }}^{\text {new }} P_{i}^{M}(h, t)\right){ }^{\text {dis }} \bar{\omega}_{i}^{M} .
\end{aligned}
$$

Adding up by gender ${ }_{\text {dis }}^{n e w} P_{i}(t)={ }_{\text {dis }}^{n e w} P_{i}^{F}(t)+{ }_{\text {dis }}^{\text {new }} P_{i}^{M}(t) \sqrt{37}$

The total of disabled retirees of age $i$ in year $t$ is ${ }_{d i s} P_{i}(t)={ }_{\text {dis }}^{\text {new }} P_{i}(t)+{ }_{\text {dis }}^{\text {old }} P_{i}(t)$. Indicating with ${ }^{i n a} \psi_{i}^{F}$ and ${ }^{i n a} \psi_{i}^{M}$ the odds of a female and a male active to have an inability, with $10 \leq h<a-26$ the new female and male retirees with inability are:

$$
{ }_{i n a}^{n e w} P_{i+1}^{F}(h, t+1)=l_{i}^{F}(h, t) \omega_{i}^{F i n a} \psi_{i}^{F}
$$

and

$$
{ }_{\text {ina }}^{\text {ina }} P_{i+1}^{M}(h, t+1)=l_{i}^{M}(h, t) \omega_{i}^{M i n a} \psi_{i}^{M} .
$$

The total correspondent of females and males, adding up by seniority is ${ }_{\text {ina }}^{\text {new }} P_{i}^{F}(t)=\sum_{h=1}^{H}$ ina $_{\text {ina }}^{\text {new }} P_{i}^{F}(h, t)$ and ${ }_{\text {ina }}^{\text {new }} P_{i}^{M}(t)=\sum_{h=1}^{H}{ }_{\text {ina }}^{\text {ina }} P_{i}^{M}(h, t)$.

Adding up by gender ${ }_{i n a}^{\text {new }} P_{i}(t)={ }_{\text {ina }}^{\text {new }} P_{i}^{F}(t)+{ }_{\text {ina }}^{\text {new }} P_{i}^{M}(t)$.

The odds of surviving of an inability retiree for the next year are ${ }^{i n a} \bar{\omega}_{i}^{F}$ for females and ${ }^{i n a} \bar{\omega}_{i}^{M}$ for males.

Therefore $\left.{ }_{\text {ina }}^{\text {old }} P_{i+1}^{F}(h, t+1)={ }_{\text {ina }}^{\text {old }} P_{i}^{F}(h, t)+{ }_{\text {ina }}^{\text {new }} P_{i}^{F}(h, t)\right){ }^{\text {ina }} \bar{\omega}_{i}^{F}$ and ${ }_{\text {ina }}^{\text {old }} P_{i+1}^{M}(h, t+1)=\left({ }_{\text {ina }}^{\text {old }} P_{i}^{M}(h, t)+{ }_{\text {ina }}^{\text {new }} P_{i}^{M}(h, t)\right){ }^{\text {ina }} \bar{\omega}_{i}^{M}$.

Adding up by gender ${ }_{i \text { ina }}^{\text {new }} P_{i}(t)={ }_{\text {ina }}^{\text {new }} P_{i}^{F}(t)+{ }_{\text {ina }}^{\text {new }} P_{i}^{M}(t) \sqrt{38}$

The total of inability retirees of age $i$ in year $t$ is ${ }_{\text {ina }} P_{i}(t)={ }_{\text {ina }}^{\text {new }} P_{i}(t)+{ }_{\text {ina }}^{\text {old }} P_{i}(t)$.

\subsubsection{Survival retirees}

The model has been made easier, supposing that the surviving spouses do not get married again. The simplification has been applied both to pensions from active who dies (called indirect) and to ones from retiree who dies (called reversibility). We specify that statistics highlight that survival probabilities of a widower are lower than in the whole male population ${ }^{39}$. Although the same difference does not notice in equal measure for female population, therefore the

\footnotetext{
${ }^{37}$ In CNPADC, disability retirees, even when they reach the age requirement for access to the pension, remain disability retirees.

${ }^{38}$ In CNPADC, inability retirees, even when they reach the retirement age, are still retirees with inability.

39 Cfr. [9].
} 
survival probability of a widow is the same of all female population, some recent researches show a change also in the widow's survival probability due to the so-called Penelope syndrom $\AA^{40}$

2.2.4.1 Indirect retirees The condition to have a pension from an active that dies is $h \geq 5 \sqrt{41}$ that, if not respected, provides for the refunding to heirs of subjective contributions paid.

Called $k$ the age of the widow(er), ${ }_{k}^{w i d} \theta_{i}^{F}$ is the probability that a female active of age $i$ leaves a widower of age $k$ and ${ }_{k}^{w i d} \theta_{i}^{M}$ is the probability that a male active of age $i$ leaves a widow of age $k$, male and female indirect retirees are: ${ }_{i n d}^{n e w} P_{k}^{M}(h, t)=\sum_{i=31}^{a-1} l_{i}^{F}(h, t-1)\left(1-\omega_{i}^{F}\right)_{k}^{\text {wid }} \theta_{i}^{F}$ and ${ }_{\text {ind }}^{\text {new }} P_{k}^{F}(h, t)=$ $\sum_{i=31}^{a-1} l_{i}^{M}(h, t-1)\left(1-\omega_{i}^{M}\right)_{k}^{w i d} \theta_{i}^{M}$.

Adding up by gender ${ }_{\text {ind }}^{n e w} P_{k}(h, t)={ }_{\text {ind }}^{n e w} P_{k}^{F}(h, t)+{ }_{\text {ind }}^{n e w} P_{k}^{M}(h, t)$.

Adding up by contributory seniority of assignor ${ }_{\text {ind }}^{\text {new }} P_{k}^{F}(t)=\sum_{h=5}^{H}{ }_{\text {ind }}^{\text {new }} P_{k}^{F}(h, t)$, ${ }_{\text {ind }}^{\text {new }} P_{k}^{M}(t)=\sum_{h=5}^{H}{ }_{\text {ind }}^{\text {new }} P_{k}^{M}(h, t)$, so ${ }_{\text {ind }}^{\text {new }} P_{k}(t)={ }_{\text {ind }}^{n e w} P_{k}^{F}(t)+{ }_{\text {ind }}^{n e w} P_{k}^{M}(t)$.

If $h<5$, it is necessary to consider that the number of indirect retirees does not increase (and so it is 0 ) but at the same time we should insert the returns for heirs from statute. In the following, we indicate the amount of contributions to be returned to the heirs of an accountant who dies without having completed the requirement for the indirect pension:

$$
\begin{aligned}
R C_{k}^{F}(h, t) & =\sum_{i=26}^{a-1} l_{i}^{M}(h, t-1)\left(1-\omega_{i}^{M}\right)_{k}^{w i d} \theta_{i}^{M} ; \\
R C_{k}^{M}(h, t) & =\sum_{i=26}^{a-1} l_{i}^{F}(h, t-1)\left(1-\omega_{i}^{F}\right)_{k}^{w i d} \theta_{i}^{F} .
\end{aligned}
$$

Adding up by contributory seniority of assignor:

$$
R C_{k}^{F}(t)=\sum_{h=1}^{H} R C_{k}^{F}(h, t) ; R C_{k}^{M}(t)=\sum_{h=1}^{H} R C_{k}^{M}(h, t) .
$$

The total of returns to heirs of age $k$ in year $t$ is $R C_{k}(t)=R C_{k}^{F}(t)+R C_{k}^{M}(t)$. It is clear that this is not a dynamic therefore for each year these amounts are counted and liquidated without any need to reconsider them at the following instants.

Denoting by ${ }^{\text {wid }} \omega_{k}^{F}$ and with ${ }^{\text {wid }} \omega_{k}^{M}$ the survival probability of widows' and widowers' accountant who dies, old indirect retirees are:

$$
{ }_{\text {ind }}^{\text {old }} P_{k+1}^{F}(t+1)=\left({ }_{\text {ind }}^{\text {old }} P_{k}^{F}(t)+{ }_{\text {ind }}^{n e w} P_{k}^{F}(t)\right)^{\text {wid }} \omega_{k}^{F}
$$

\footnotetext{
40 According to some studies Penelope syndrome involves one over 75 years old woman every five, they die because they feel alone and sad - http://www.medicitalia.it/blog/psicologia/4580-sindrome-penelope-donne-malate-attesa-affetti-legami.html .

41 Although CNPADC provides different requirements for income and DC pensions, respectively 10 and 5 years, this paper considers the minimum contribution of 5 years because of the latter represent the future of the Fund.
} 


$$
{ }_{\text {ind }}^{\text {old }} P_{k+1}^{M}(t+1)=\left({ }_{\text {ind }}^{\text {old }} P_{k}^{M}(t)+{ }_{\text {ind }}^{\text {new }} P_{k}^{M}(t)\right){ }^{\text {wid }} \omega_{k}^{M} .
$$

The total of old indirect retirees is ${ }_{i n d}^{\text {old }} P_{k}(t)={ }_{\text {ind }}^{\text {old }} P_{k}^{F}(t)+{ }_{\text {ind }}^{\text {old }} P_{k}^{M}(t)$.

The total of indirect retirees of age $k$ in year $t$ is

${ }_{\text {ind }} P_{k}(t)={ }_{\text {ind }}^{\text {new }} P_{k}(t)+{ }_{\text {ind }}^{\text {old }} P_{k}(t)$.

2.2.4.2 Reversibility retirees The new survival retirees, with maximum possible age 105, are:

$$
\begin{aligned}
& { }_{\text {rev }}^{\text {new }} P_{k}^{F}(h, t)=\sum_{i=a}^{104} \operatorname{dir}_{i} P_{i}^{M}(h, t-1)\left(1-\omega_{i}^{M}\right)_{k}^{w i d} \theta_{i}^{M} ; \\
& { }_{\text {rev }}^{\text {new }} P_{k}^{M}(h, t)=\sum_{i=a}^{104} \operatorname{dir} P_{i}^{F}(h, t-1)\left(1-\omega_{i}^{F}\right)_{k}^{w i d} \theta_{i}^{F} .
\end{aligned}
$$

Adding up by gender we obtain ${ }_{\text {rev }}^{\text {new }} P_{k}(h, t)={ }_{\text {rew }}^{\text {new }} P_{k}^{F}(h, t)+{ }_{\text {rev }}^{\text {new }} P_{k}^{M}(h, t)$.

Adding up to the seniority of assignor, we have:

$$
{ }_{\text {new }}^{\text {new }} P_{k}^{F}(t)=\sum_{h=1}^{H} \text { rew } P_{k}^{F}(h, t) \text { and }{ }_{r e v}^{\text {new }} P_{k}^{M}(t)=\sum_{h=1}^{H} \text { rew }{ }_{\text {rev }}^{\text {new }} P_{k}^{M}(h, t)
$$

therefore ${ }_{\text {rev }}^{\text {new }} P_{k}(t)={ }_{\text {rew }}^{\text {new }} P_{k}^{F}(t)+{ }_{\text {rev }}^{\text {new }} P_{k}^{M}(t)$.

The old reversibility retirees, recursively, are:

$$
\begin{aligned}
& { }_{\text {rev }}^{\text {old }} P_{k+1}^{F}(t+1)=\left({ }_{\text {rev }}^{\text {old }} P_{k}^{F}(t)+{ }_{\text {rev }}^{\text {new }} P_{k}^{F}(t)\right){ }^{\text {wid }} \omega_{k}^{F} \text { and } \\
& { }_{\text {rev }}^{\text {old }} P_{k+1}^{M}(t+1)=\left({ }_{\text {rev }}^{\text {old }} P_{k}^{M}(t)+{ }_{\text {rev }}^{\text {new }} P_{k}^{M}(t)\right){ }^{\text {wid }} \omega_{k}^{M} \text {. }
\end{aligned}
$$

The total of old ones is ${ }_{\text {rev }}^{\text {old }} P_{k}(t)={ }_{\text {rev }}^{\text {old }} P_{k}^{F}(t)+{ }_{\text {rev }}^{\text {old }} P_{k}^{M}(t)$.

The total of reversibility retirees of age $k$ in year $t$ is so given by

$$
{ }_{\text {rev }} P_{k}(t)={ }_{\text {rev }}^{\text {new }} P_{k}(t)+{ }_{\text {rev }}^{\text {old }} P_{k}(t) .
$$

The total of retirees is equal to the sum of all cases considered above:

$$
P(t)={ }_{d i r} P_{i}(t)+{ }_{d i s} P_{i}(t)+{ }_{i n a} P_{i}(t)+{ }_{i n d} P_{k}(t)+{ }_{r e v} P_{k}(t) .
$$

\subsection{Economic and financial technical bases}

Once defined the number of each type of pension, the model of $2012^{42}$ allows to determine the amount of pensions and income on the basis of the new rules indicated in the Cazzola-Treu proposal to formalize the resulting PAYG DC rate of tendential equilibrium.

By virtue of the indications deriving from the law proposal the problem is to highlight what are the variables that influence the two components of mandatory pension benefit. The intent is to work on these variables to guarantee a substitution rate on direct pension not less than $60 \%$, and this percentage inevitably drags on survival pension.

Indicating with $\bar{R}_{i}(h, t)$ the average gross income of an accountant of age $i$ in year $t$, with $h$ years of seniority and with $s{ }^{43}$ the subjective contribution rate, which is now from $12 \%$ to $100 \%$. Each member can evaluate his own

42 Cfr. [5].

43 In the model the rate is not that one of the law proposal but that one of CNPADC. 
situation using the fund simulator; vice versa, it is not possible to obtain a systemic evaluation with regard to the adequacy and this is the contribution of this paper.

In particular, the applied rate is equal to $\phi=S_{c}+B\left(1-\frac{h}{H}\right)$ where $S_{c}$ is the paid subjective contribution rate, $B$ the plus given based on the rate deposited, $H$ the maximum contribution seniority in CNPADC, $h$ the seniority of contribution's accountant and $\frac{h}{H}$ the intergenerational equity coefficient.

The total revenues in $t$ is $T(t)=\sum_{i, h}\left(l_{i}^{F}(h, t)+l_{i}^{M}(h, t)\right) \bar{R}_{i}(h, t)$, from which it is possible to calculate the amount of contributions

$C(t)=\left(S_{c}+B\left(1-\frac{h}{H}\right)\right) T(t)=\left(S_{c}+B\left(1-\frac{h}{H}\right)\right) \sum_{i, h}\left(l_{i}^{F}(h, t)+l_{i}^{M}(h, t)\right) \bar{R}_{i}(h, t)$.

Indicating with $c_{i}(t)$ the transformation coefficients for age $i$ in year $t, r_{z}$ the annual capitalization rate calculated as five-year geometrical mean of nominal GDP in year $z$, the average pension of a retiree from year $t$, after $h$ years of contributory seniority and zero years of seniority in pension, hypothesizing that contributions are evaluated at the year end and pensions paid at the beginning of the year, is:

- for direct pensions:

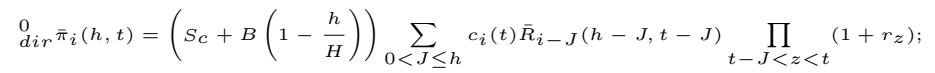

- for disability pensions:

$$
{ }_{d i s}^{0} \bar{\pi}_{i}(h, t)=\left(S_{c}+B\left(1-\frac{h}{H}\right)\right) \underset{0<J \leq h}{\sum_{i}(t) \bar{R}_{i-J}(h-J, t-J)} \prod_{t-J<z<t}\left(1+r_{z}\right) ;
$$

- for inability pensions:

$$
{ }_{i n a}^{0} \bar{\pi}_{i}(h, t)=\left(S_{C}+B\left(1-\frac{h}{H}\right)\right) \sum_{0<J \leq h} c_{i}(t) \bar{R}_{i-J}(h-J, t-J) \prod_{t-J<z<t}\left(1+r_{z}\right) ;
$$

- for indirect pensions:

if $h \geq 5$,

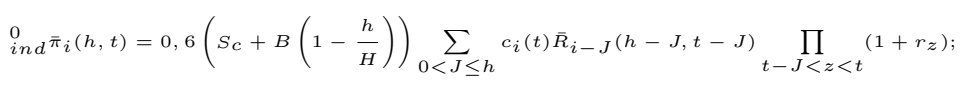

if $h<5$,

$$
{ }_{i n d}^{0} \overline{Q C}_{i}(h, t)=\left(S_{c}+B\left(1-\frac{h}{H}\right)\right) \sum_{0<J \leq h} \bar{R}_{i-J}(h-J, t-J) \prod_{t-J<z<t}\left(1+r_{z}\right),
$$

considering that in case of indirect pensions with contribution lower than 5 years, deposited contributes are given back to survivors, we suppose that latter are equal to the product between contribution rate, contributory seniority and average earnings $\bar{R}_{i-J}(h-J, t-J)$ of the assignor;

- for reversibility pensions:

$$
{ }_{\text {rev }}^{0} \bar{\pi}_{i}(h, t)=0,6_{d i r}^{v} \bar{\pi}_{i}(h, t) .
$$


If $r_{z}^{\prime}$ is the appreciation rate of pensions in year $z, v(v \geq 0)$ the number of years in pension, we have:

- direct:

$$
\begin{aligned}
& { }_{d i r}^{v} \bar{\pi}_{i}(h, t)= \\
& \left(\left(S_{c}+B\left(1-\frac{h}{H}\right)\right)\left(\sum_{0<J \leq h} c_{i-v}(t) \bar{R}_{i-v-J}(h-J, t-J) \prod_{t-J<z<t}\left(1+r_{z}\right)\right)\right) \prod_{t-v<z \leq t}\left(1+r_{z}^{\prime}\right) ;
\end{aligned}
$$

- disability:

$$
\begin{aligned}
& \underset{d i s}{v} \bar{\pi}_{i}(h, t)= \\
& \left(\left(S_{c}+B\left(1-\frac{h}{H}\right)\right)\left(\sum_{0<J \leq h} c_{i-v}(t) \bar{R}_{i-v-J}(h-J, t-J) \prod_{t-J<z<t}\left(1+r_{z}\right)\right)\right) \prod_{t-v<z \leq t}\left(1+r_{z}^{\prime}\right) ;
\end{aligned}
$$

- inability:

$$
\begin{aligned}
& \stackrel{v}{\text { ina }} \overline{\bar{\pi}}_{i}(h, t)= \\
& \left(\left(S_{c}+B\left(1-\frac{h}{H}\right)\right)\left(\sum_{0<J \leq h} c_{i-v}(t) \bar{R}_{i-v-J}(h-J, t-J) \prod_{t-J<z<t}\left(1+r_{z}\right)\right)\right) \prod_{t-v<z \leq t}\left(1+r_{z}^{\prime}\right) ;
\end{aligned}
$$

- indirect:

if $h \geq 5$,

$$
\begin{aligned}
& { }_{\text {ind }}^{v} \bar{\pi}_{i}(h, t)= \\
& \left(0,6\left(S_{c}+B\left(1-\frac{h}{H}\right)\right)\left(\sum_{0<J \leq h} c_{i-v}(t) \bar{R}_{i-v-J}(h-J, t-J) \prod_{t-J<z<t}\left(1+r_{z}\right)\right)\right) \prod_{t-v<z \leq t}\left(1+r_{z}^{\prime}\right) ;
\end{aligned}
$$

if $h<5$,

$$
{ }_{i n d}^{0} \overline{Q C}_{i}(h, t)=\left(S_{c}+B\left(1-\frac{h}{H}\right)\right) \sum_{0<J \leq h} \bar{R}_{i-v-J}(h-J, t-J) \prod_{t-J<z<t}\left(1+r_{z}\right),
$$

considering, as above, that in case of indirect pensions with contribution lower than 5 years, deposited contributes are given back to survivors, we suppose that latter are equal to the product between contribution rate, contributory seniority and average earnings $\bar{R}_{i-v-J}(h-J, t-J)$ of the assignor;

- reversibility:

$$
{ }_{r e v}^{v} \bar{\pi}_{i}(h, t)={ }_{r e v}^{0} \bar{\pi}_{i}(h, t) \prod_{t-v<z \leq t}\left(1+r_{z}^{\prime}\right)
$$

The number of female and male retirees with parameters defined above is:

- direct:

$$
\underset{d i r}{v} P_{i}^{F}(h, t)={ }_{d i r}^{0} P_{i-v}^{F}(h, t-v) \prod_{j=1-v}^{i-1} \omega_{j}^{F}, \underset{d i r}{v} P_{i}^{M}(h, t)={ }_{d i r}^{0} P_{i-v}^{M}(h, t-v) \prod_{j=1-v}^{i-1} \omega_{j}^{M}
$$


- disability:

$$
\underset{d i s}{v} P_{i}^{F}(h, t)={ }_{d i s}^{0} P_{i-v}^{F}(h, t-v) \prod_{j=1-v}^{i-1} d i s \omega_{j}^{F}, \underset{d i s}{v} P_{i}^{M}(h, t)={ }_{d i s}^{0} P_{i-v}^{M}(h, t-v) \prod_{j=1-v}^{i-1} d i s \omega_{j}^{M} ;
$$

- inability:

$$
{ }_{i n a}^{v} P_{i}^{F}(h, t)={ }_{i n a}^{0} P_{i-v}^{F}(h, t-v) \prod_{j=1-v}^{i-1}{ }_{i n a} \omega_{j}^{F},{ }_{i n a} P_{i}^{M}(h, t)={ }_{i n a}^{0} P_{i-v}^{M}(h, t-v) \prod_{j=1-v}^{i-1}{ }^{i n a} \omega_{j}^{M} ;
$$

- indirect:

if $h \geq 5$,

$$
{ }_{i n d}^{v} P_{i}^{F}(h, t)={ }_{i n d}^{0} P_{i-v}^{F}(h, t-v) \prod_{j=1-v}^{i-1} w i d \omega_{j}^{F} ; i_{i n d} P_{i}^{M}(h, t)={ }_{i n d}^{0} P_{i-v}^{M}(h, t-v) \prod_{j=1-v}^{i-1} w i d \omega_{j}^{M} ;
$$

if $h<5$,

$$
v_{R C_{i}^{M}}(h, t)=\sum_{i=26}^{a-1} l_{i}^{F}(h, t-1)\left(1-\omega_{i}^{F}\right)_{k}^{w i d} \theta_{i}^{F} ; v_{R C_{i}^{F}}(h, t)=\sum_{i=26}^{a-1} l_{i}^{M}(h, t-1)\left(1-\omega_{i}^{M}\right)_{k}^{w i d} \theta_{i}^{M}{ }^{M}
$$

- reversibility:

$$
{ }_{r e v}^{v} P_{i}^{F}(h, t)=r_{r e v}^{0} P_{i-v}^{F}(h, t-v) \prod_{j=1-v}^{i-1} w_{i d} \omega_{j}^{F}, r_{r e v} P_{i}^{M}(h, t)={ }_{r e v}^{0} P_{i-v}^{M}(h, t-v) \prod_{j=1-v}^{i-1} w i d \omega_{j}^{M} .
$$

The total of pensions in $t$ is:

- direct: ${ }_{d i r}^{v} \pi_{i}(h, t)={ }_{d i r}^{v} \bar{\pi}_{i}(h, t)\left({ }_{d i r}^{v} P_{i}^{F}(h, t)+{ }_{d i r}^{v} P_{i}^{M}(h, t)\right)$;

- disability: ${ }_{d i s}^{v} \pi_{i}(h, t)={ }_{d i s}^{v} \bar{\pi}_{i}(h, t)\left({ }_{d i s}^{v} P_{i}^{F}(h, t)+{ }_{d i s}^{v} P_{i}^{M}(h, t)\right)$;

- inability: ${ }_{i n a}^{v} \pi_{i}(h, t)={ }_{i n a}^{v} \bar{\pi}_{i}(h, t)\left({ }_{i n a}^{v} P_{i}^{F}(h, t)+{ }_{i n a}^{v} P_{i}^{M}(h, t)\right)$;

- indirect:

- if $h \geq 5$,

$$
{ }_{\text {ind }}^{v} \pi_{i}(h, t)={ }_{i n d}^{v} \bar{\pi}_{i}(h, t)\left({ }_{i n d}^{v} P_{i}^{F}(h, t)+{ }_{i n d}^{v} P_{i}^{M}(h, t)\right) ;
$$

- if $h<5$,

$$
{ }_{\text {ind }}^{v} Q C_{i}(h, t)={ }_{\text {ind }}^{v} \overline{Q C}_{i}(h, t)\left({ }^{v} R C_{i}^{F}(h, t)+{ }^{v} R C_{i}^{M}(h, t)\right) ;
$$

- reversibility: ${ }_{\text {rev }}^{v} \pi_{i}(h, t)={ }_{r e v}^{v} \bar{\pi}_{i}(h, t)\left({ }_{\text {rev }}^{v} P_{i}^{F}(h, t)+{ }_{\text {rev }}^{v} P_{i}^{M}(h, t)\right)$.

Supposing $r_{z}$ and $r_{z}^{\prime}$ self-supporting by $z, \bar{R}_{i}(h, 0)=d_{i, h}, \bar{R}_{i}(h, t)=d_{i, h}(1+r)^{t}$ adding up to ages, seniority in pension and contributory seniority, we have:

\footnotetext{
${ }^{44}$ As explained, we are not in front of a dynamics because in each year the number of return is counted and paid off with no needs of reconsidering it in the following moments.
} 
- direct:

$$
\begin{aligned}
& \pi_{d i r}(t)= \\
& \sum_{i=61}^{104} \sum_{v=0}^{i-61} \sum_{h=25}^{H}\left(S_{c}+B\left(1-\frac{h}{H}\right)\right)\left(\left(1+r^{\prime}\right)^{v} \sum_{0<J \leq h} c_{i}(t) d_{i-v-J, h-j}(1+r)^{t-J}(1+r)^{J-1}\right) . \\
& \left({ }_{d i r}^{0} P_{i-v}^{F}(h, t-v) \prod_{j=i-v}^{i-1} \omega_{j}^{F}+{ }_{d i r}^{0} P_{i-v}^{M}(h, t-v) \prod_{j=i-v}^{i-1} \omega_{j}^{M}\right)
\end{aligned}
$$

- disability:

$$
\begin{aligned}
& \sum_{i=26}^{\pi_{d i s}(t)=} \sum_{v=36}^{a-1} \sum_{h=10}^{i-61}\left(S_{c}+B\left(1-\frac{h}{H}\right)\right)\left(\left(1+r^{\prime}\right)^{v} \sum_{0<J \leq h} c_{i}(t) d_{i-v-J, h}(1+r)^{t-J}(1+r)^{J-1}\right) \\
& \cdot\left(\begin{array}{l}
0 \\
d i s
\end{array} P_{i-v}^{F}(h, t-v) \prod_{j=i-v}^{i-1} d i s \omega_{j}^{F}+{ }_{d i s}^{0} P_{i-v}^{M}(h, t-v) \prod_{j=i-v}^{i-1} d i s \omega_{j}^{M}\right)
\end{aligned}
$$

- inability:

$$
\begin{aligned}
& \pi_{\text {ina }}(t)= \\
& \sum_{i=26}^{a-1} \sum_{\nu=36}^{i-61} \sum_{h=10}^{H}\left(S_{c}+B\left(1-\frac{h}{H}\right)\right)\left(\left(1+r^{\prime}\right)^{v} \sum_{0<J \leq h} c_{i}(t) d_{i-v-J, h}(1+q)^{t-J}(1+r)^{J-1}\right) . \\
& \left({ }_{i n a}^{0} P_{i-v}^{F}(h, v) \prod_{j=i-v}^{i-1} i n a \omega_{j}^{F}+{ }_{i n a}^{0} P_{i-v}^{M}(h, v) \prod_{j=i-v}^{i-1} i n a \omega_{j}^{M}\right)
\end{aligned}
$$

- indirect:

$$
\begin{aligned}
& \pi_{\text {ind }}(t)= \\
& \sum_{i=31}^{a-1} \sum_{v=0}^{i-61} \sum_{h=5}^{H}\left(S_{c}+B\left(1-\frac{h}{H}\right)\right)\left(\left(1+r^{\prime}\right)^{v} \sum_{0<J \leq h} c_{i}(t) d_{i-v-J, h}(1+q)^{t-J}(1+r)^{J-1}\right) \\
& \cdot\left(\begin{array}{l}
0 \\
i n d
\end{array} P_{i-v}^{F}(h, \nu) \prod_{j=i-v}^{i-1} w i d \omega_{j}^{F}+{ }_{i n d}^{0} P_{i-v}^{M}(h, v) \prod_{j=i-v}^{i-1} w i d \omega_{j}^{M}\right)+ \\
& +\sum_{i=26}^{a-1} \sum_{v=31}^{i-61} \sum_{\substack{h=1 \\
h \neq 5}}^{5}\left(S c+B\left(1-\frac{h}{H}\right)\right)\left(\sum_{0<J \leq h} d_{i-v-J, h}(1+q)^{t-J}(1+r)^{J-1}\right) \\
& \cdot(\underbrace{v}_{i n d} R_{i-v}^{F}(h, v)+{ }_{i n d}^{v} R_{i-v}^{M}(h, v))
\end{aligned}
$$

- reversibility:

$$
\begin{gathered}
\pi_{r e v}(t)=\sum_{i=61}^{104} \sum_{v=0}^{i-61} \sum_{h=5}^{H}\left(S_{c}+B\left(1-\frac{h}{H}\right)\right)\left({ }_{r e v}^{0} \bar{\pi}_{i}(h, t)\left(1+r^{\prime}\right)^{v}\right) . \\
\cdot\left({ }_{r e v}^{0} P_{i-v}^{F}(h, v) \prod_{j=i-v}^{i-1}{ }^{w i d} \omega_{j}^{F}+{ }_{r e v}^{0} P_{i-v}^{M}(h, v) \prod_{j=i-v}^{i-1}{ }^{w i d} \omega_{j}^{M}\right)
\end{gathered}
$$


The total of DC pensions in year $t$ is:

$$
\pi_{d c}(t)=\pi_{d i r}(t)+\pi_{d i s}(t)+\pi_{i n a}(t)+\pi_{i n d}(t)+\pi_{r e v}(t) .
$$

After having verified the contribution part of the compulsory pension, it is necessary to insert a fix portion of the pension $\pi_{f i x}(t)$ provided by the CazzolaTreu proposal, called from now fp. For the basic public system it is funded through general taxation. Considering that CNPADC does not receive money from general taxation, it is assumed that the contribution $\eta$ equal to $1 \%$ at the expense of both active accountants and retirees of CNPADC. Alternatively, it would be possible to draw from integrative contribution equal to $4 \%$, applied to all revenues of the previous year 45 In order to determine the fp amount for the members of CNPADC, for the sake of simplicity, the social allowance has been used.

The assumption to cover the fp has been verified considering the active members of CNPADC, the active retirees, the inactive retirees and average accountant's revenues and considering that in Italy the social allowance amount is equal to $€ 453$ per month.

The number of female and male retirees, of age $i$ and retirement seniority $v$, for fp is:

- ${ }^{v} P_{i}^{F}(t)=\sum_{h=10}^{H}\left({ }_{d i r}^{v} P_{i}^{F}(h, t)+{ }_{d i s}^{v} P_{i}^{F}(h, t)+{ }_{i n a}^{v} P_{i}^{F}(h, t)+{ }_{i n d}^{v} P_{i}^{F}(h, t)+{ }_{r e v}^{v} P_{i}^{F}(h, t)\right)$;

- ${ }^{v} P_{i}^{M}(t)=\sum_{h=10}^{H}\left({ }_{d i r}^{v} P_{i}^{M}(h, t)+{ }_{d i s}^{v} P_{i}^{M}(h, t)+{ }_{i n a}^{v} P_{i}^{M}+{ }_{i n d}^{v} P_{i}^{M}(h, t)+{ }_{r e v}^{v} P_{i}^{M}(h, t)\right)$.

Defining $F(t)$ the fp amount of the year $(t=0,1,2, \ldots), t=0$ the conventional reference year, $\delta_{y}$ the appreciation rate of the fixed pension amount in year $y$, adding up to $i$ and $v$, we obtain the fp:

$$
\begin{aligned}
\pi_{f i x}(t)= & F(0) \prod_{y=1}^{t}\left(1+\delta_{y}\right) \sum_{i=61}^{\Omega-1} \sum_{v=0}^{\Omega-61}\left({ }^{v} P_{i}^{F}(t)+{ }^{v} P_{i}^{M}(t)\right)= \\
& =F(t) \sum_{i=61}^{\Omega-1} \sum_{v=0}^{\Omega-61}\left({ }^{v} P_{i}^{F}(t)+{ }^{v} P_{i}^{M}(t)\right) .
\end{aligned}
$$

The total amount of the pension is $\pi(t)=\pi_{d c}(t)+\pi_{f i x}(t)$.

\subsection{Contribution rate at a tendential equilibrium in a pay- as-you-go system}

The limit between all the pension voices (dc and fix) and the total amount of net income earned it is a datum value $A$ called contribution rate at a tendential equilibrium in a PAYG pension system.

As said before $\phi=S_{c}+B\left(1-\frac{h}{H}\right),(1-\phi) T$ are the net income, so it is possible to write in the demographic equilibrium: $\frac{1}{1-\phi}\left(\frac{\pi_{d c}+\pi_{f i x}}{T}\right)_{\infty}=A$.

\footnotetext{
45 As reported in the actuarial balance.
} 
Fixing $\lim _{t \rightarrow \infty} \frac{\pi(t)}{T(t)}=\left(\frac{\pi}{T}\right)_{\infty}$, in a compact way

$$
\frac{1}{1-\phi}\left(\frac{\pi}{T}\right)_{\infty}=A
$$

The substitution rate for the direct pension is fixed at least at $60 \%$ as indicated in the Cazzola-Treu law proposal and the contribution rate $\phi$ is that one currently adopted by CNPADC as the minimum.

In the model we want to demonstrate the existence of the limit proving the existence of all component limits.

Transforming the ratio $\left(\frac{\pi}{T}\right)_{\infty}$ for the demographic equilibrium, dividing by the eigenvalue $\lambda_{0}^{t}$, we can calculate the limit, for which the existence is ensured applying the Theorem 1 , as follows

$$
\left(\frac{\pi}{T}\right)_{\infty}=\left(\frac{\frac{\pi(t)}{\lambda_{0}^{t}}}{\frac{T(t)}{\lambda_{0}^{t}}}\right)_{\infty}=A(1-\phi) .
$$

Transforming through (1) we obtain $\left(\frac{\pi(t)}{\lambda_{0}^{t}}\right)_{\infty}=A(1-\phi)\left(\frac{T(t)}{\lambda_{0}^{t}}\right)_{\infty}$.

$\phi$ could be the same for ever or it would be changed by the accountant.

The application of Theorem 1 is subordinated to the construction of the expression of the number of retirees, for each category, depending on actives.

\subsubsection{For direct pensions}

For females and males of age $i$ with $26<i<a$, the rate of first registration to CNPADC is supposed to be $\tau_{i}^{F}(t)$ for females and $\tau_{i}^{M}(t)$ for males.

Considering the Hadamard product between the vector $\tau^{F}(t)=\left(\tau_{0}^{F}(t), \tau_{1}^{F}(t), \ldots, \tau_{\Omega-1}^{F}(t)\right)$ and the population state vector $x^{F}(t)$ and between $\tau^{M}(t)=\left(\tau_{0}^{M}(t), \tau_{1}^{M}(t), \ldots, \tau_{\Omega-1}^{M}(t)\right)$ and $x^{M}(t)$, we obtain the vector of new members (females and males) in year $t$, that is $l^{F}(t)=x^{F}(t) \circ \tau^{F}(t)$ and $l^{M}(t)=x^{M}(t) \circ \tau^{M}(t)$.

Assuming that the rate of first registration to CNPADC is stable over time for each $26<i<61,10 \leq h \leq i-26, t \geq h$, we have for females and males:

$$
\begin{aligned}
& l_{i}^{F}(h, t)=\tau_{i-h}^{F} x_{i-h}^{F}(t-h) \omega_{i-h}^{F} \omega_{i-h+1}^{F} \cdots \omega_{i-1}^{F}=\tau_{i-h}^{F} x_{i}^{F}(t) ; \\
& l_{i}^{M}(h, t)=\tau_{i-h}^{M} x_{i-h}^{M}(t-h) \omega_{i-h}^{M} \omega_{i-h+1}^{M} \cdots \omega_{i-1}^{M}=\tau_{i-h}^{M} x_{i}^{M}(t) .
\end{aligned}
$$

Applying the Theorem 1 we have for females and males:

$$
\lim _{t \rightarrow+\infty} \frac{l_{i}^{F}(h, t)}{\lambda_{0}^{t}}=\tau_{i-h}^{F} s x_{i}^{0 F}, \lim _{t \rightarrow+\infty} \frac{l_{i}^{M}(h, t)}{\lambda_{0}^{t}}=\tau_{i-h}^{M} s x_{i}^{0 M} .
$$

With the same hypotheses $61 \leq i \leq a, 10 \leq h \leq i-26$, assuming that the retirement rate of actives with seniority $h$ is $\sigma_{i}^{F}(h), \sigma_{i}^{M}(h)$, it is possible to calculate the retirees as follows:

$$
P_{i}^{F}(h, t)=\sigma_{i-1}^{F}(h) l_{i-1}^{F}(h, t-1) \omega_{i-1}^{F}, P_{i}^{M}(h, t)=\sigma_{i-1}^{M}(h) l_{i-1}^{M}(h, t-1) \omega_{i-1}^{M} .
$$


For females, we have:

$$
\begin{gathered}
P_{62}^{F}(h, t)=\sigma_{61}^{F}(h) l_{61}^{F}(h, t-1) \omega_{61}^{F}=\sigma_{61}^{F}(h) \tau_{61-h}^{F} x_{61}^{F}(t-1) \omega_{61}^{F}= \\
\sigma_{61}^{F}(h) \tau_{61-h}^{F} x_{62}^{F}(t) ; \\
P_{63}^{F}(h, t)=\sigma_{62}^{F}(h-1) l_{62}^{F}(h-1, t-1) \omega_{62}^{F}=\sigma_{62}^{F}(h-1) l_{61}^{F}(h-2, t-2) \omega_{62}^{F} \omega_{63}^{F}= \\
=\sigma_{62}^{F}(h-1) \tau_{62-(h-2)}^{F} x_{61}^{F}(t-2) \omega_{61}^{F} \omega_{62}^{F}=\sigma_{62}^{F}(h-1) \tau_{62-(h-2)}^{F} x_{63}^{F}(t) ; \\
\vdots \\
P_{70}^{F}(h, t)=\sigma_{69}^{F}(h-8) \tau_{64-(h-9)}^{F} x_{70}^{F}(t) .
\end{gathered}
$$

For males, we have:

$$
\begin{gathered}
P_{62}^{M}(h, t)=\sigma_{61}^{M}(h) l_{61}^{M}(h, t-1) \omega_{61}^{M}=\sigma_{61}^{M}(h) \tau_{61-h}^{M} x_{61}^{M}(t-1) \omega_{61}^{M}= \\
\sigma_{61}^{M}(h) \tau_{61-h}^{M} x_{62}^{M}(t) ; \\
P_{63}^{M}(h, t)=\sigma_{62}^{M}(h-1) l_{62}^{M}(h-1, t-1) \omega_{62}^{M}=\sigma_{62}^{M}(h-1) l_{61}^{M}(h-2, t-2) \omega_{62}^{M} \omega_{63}^{M}= \\
=\sigma_{62}^{M}(h-1) \tau_{62-(h-2)}^{M} x_{61}^{M}(t-2) \omega_{61}^{M} \omega_{62}^{M}=\sigma_{62}^{M}(h-1) \tau_{62-(h-2)}^{M} x_{63}^{M}(t) ; \\
\vdots \\
P_{70}^{M}(h, t)=\sigma_{69}^{M}(h-8) \tau_{64-(h-9)}^{M} x_{70}^{M}(t) .
\end{gathered}
$$

Therefore, the new retirees of age $i$ are proportional to the $i$-th component of population vector. Dividing by the $t$-th power of the eigenvalue and passing to the limit, we obtain for females and males, respectively:

$$
\begin{aligned}
& \lim _{t \rightarrow+\infty} \frac{P_{i}^{F}(h, t)}{\lambda_{0}^{t}}=\bar{\tau}_{i, h}^{F} s x_{i}^{0 F} ; \\
& \lim _{t \rightarrow+\infty} \frac{P_{i}^{M}(h, t)}{\lambda_{0}^{t}}=\bar{\tau}_{i, h}^{M} s x_{i}^{0 M},
\end{aligned}
$$

where $\bar{\tau}_{i, h}^{F}=\sigma_{i}^{F} \tau_{i, h}^{F}, \bar{\tau}_{i, h}^{M}=\sigma_{i}^{M} \tau_{i, h}^{M}$.

Considering that $v$ years ago, new retirees were active, we have $\lim _{t \rightarrow+\infty} \frac{P_{i-v}^{F}(h, t-v)}{\lambda_{0}^{t}}=\frac{1}{\lambda_{0}^{v}} \lim _{t \rightarrow+\infty} \frac{P_{i-v}^{F}(h, t-v)}{\lambda_{0}^{t-v}}=\frac{1}{\lambda_{0}^{v}} \bar{\tau}_{i, h}^{F} s x_{i}^{0 F} ;$ $\lim _{t \rightarrow+\infty} \frac{P_{i-v}^{M}(h, t-v)}{\lambda_{0}^{t}}=\frac{1}{\lambda_{0}^{v}} \lim _{t \rightarrow+\infty} \frac{P_{i-v}^{M}(h, t-v)}{\lambda_{0}^{t-v}}=\frac{1}{\lambda_{0}^{v}} \bar{\tau}_{i, h}^{M} s x_{i}^{0 M}$.

The limit of $\frac{\pi_{d i r}(t)}{T(t)}$ for direct pensions, using $\sqrt{4}$ and $(5)$ for the denominator and considering, for simplicity, transformation coefficients independent of age and assuming the existence of the limit $\lim _{t \rightarrow \infty} c(t)=c_{0}$, we obtain:

$$
\begin{aligned}
& \lim _{t \rightarrow \infty} \frac{\pi_{d i r}(t)}{T(t)}=\left(\frac{\pi_{d i r}}{T}\right)_{\infty}= \\
= & c_{0} \frac{\sum_{i=61}^{104} \sum_{v=0}^{i-61} \sum_{h=25}^{i-26}\left(S_{c}+B\left(1-\frac{h}{H}\right)\right) \cdot\left(\left(1+r^{\prime}\right)^{v} \sum_{0<J \leq h} d_{i-v-J, t-j}(1+r)^{-1}\right) \cdot D^{d i r}(i, v, h)}{\sum_{i, h}\left(\bar{\tau}_{i, h}^{F} s x_{i}^{0 F}+\bar{\tau}_{i, h}^{M} s x_{i}^{O M}\right) d_{i, h}}
\end{aligned}
$$


where

$$
D^{d i r}(i, v, h)=\left(\frac{1}{\lambda_{0}^{v}} \bar{\tau}_{i, h}^{F} s x_{i}^{0 F} \prod_{j=i-v}^{i-1} \omega_{j}^{F}+\frac{1}{\lambda_{0}^{v}} \bar{\tau}_{i, h}^{M} s x_{i}^{0 M} \prod_{j=i-v}^{i-1} \omega_{j}^{M}\right)
$$

\subsubsection{For disability pensions}

In case of disability for disease for all females and males of age $i$ with $26<i<a$ and $10 \leq h \leq i-26$, with ${ }^{\text {dise }} \psi_{i}^{F}$ and ${ }^{d i s e} \psi_{i}^{M}$ the odds to become invalid due to disease, for females and males respectively, we have:

${ }_{\text {dis-dise }} P_{i+1}^{F}(h, t+1)=l_{i}^{F}(h, t) \omega_{i}^{F}$ dise $\psi_{i}^{F}$
${ }_{\text {dis-dise }} P_{i+1}^{M}(h, t+1)$
(is- $l_{i}^{M}(h, t) \omega_{i}^{M \text { dise }} \psi_{i}^{M}$.

For $t$ sufficiently large, from (2) and (3) we obtain:

${ }_{\text {dis-dise }}^{0} P_{i}^{F}(h, t)=l_{i}^{F}(h, t) \omega_{i}^{F}{ }^{\text {dise }} \psi_{i}^{F}=\tau_{i-h}^{F}{ }^{\text {dise }} \psi_{i}^{F} x_{i}^{F}(t)$,

${ }_{\text {dis-dise }} P_{i}^{M}(h, t)=l_{i}^{M}(h, t) \omega_{i}^{M \text { dise }} \psi_{i}^{M}=\tau_{i-h}^{M}{ }^{\text {dise }} \psi_{i}^{M} x_{i}^{M}(t)$.

In case of disability for injury for females and males of age $i$ with $26<i<a$ and $5 \leq h \leq i-26$, with ${ }^{i n j} \psi_{i}^{F}$ and ${ }^{i n j} \psi_{i}^{M}$ the odds to become invalid due to injury, for females and males respectively, we have:

$$
\begin{aligned}
& { }_{d i s-i n j}^{0} P_{i+1}^{F}(h, t+1)=l_{i}^{F}(h, t) \omega_{i}^{F i n j} \psi_{i}^{F} ; \\
& { }_{d i s-i n j}^{0} P_{i+1}^{M}(h, t+1)=l_{i}^{M}(h, t) \omega_{i}^{M i n j} \psi_{i}^{M} .
\end{aligned}
$$

For $t$ sufficiently large, from (2) and (3) we obtain:

$$
\begin{aligned}
& { }_{d i s-i n j}^{0} P_{i}^{F}(h, t)=l_{i}^{F}(h, t) \omega_{i}^{F i n j} \psi_{i}^{F}=\tau_{i-h}^{F}{ }^{i n j} \psi_{i}^{F} x_{i}^{F}(t), \\
& { }_{d i s-i n j}^{0} P_{i}^{M}(h, t)=l_{i}^{M}(h, t) \omega_{i}^{M i n j} \psi_{i}^{M}=\tau_{i-h}^{M}{ }^{i n j} \psi_{i}^{M} x_{i}^{M}(t) .
\end{aligned}
$$

The sum of disable actives due to disease and injury is for females and males, respectively ${ }_{d i s}^{0} P_{i}^{F}(h, t)={ }_{\text {dis-dise }}^{0} P_{i}^{F}(h, t)+{ }_{\text {dis-inj }}^{0} P_{i}^{F}(h, t)$ and ${ }_{\text {dis }}^{0} P_{i}^{M}(h, t)={ }_{\text {dis-dise }}^{0} P_{i}^{M}(h, t)+{ }_{\text {dis-inj }}^{0} P_{i}^{M}(h, t)$.

Applying the Theorem 1 for females and males, considering that the probability to become disable due to disease and injury is different, we have: $\lim _{t \rightarrow+\infty} \frac{0^{0} i s P_{i}^{F}(h, t)}{\lambda_{0}^{t}}=$ $\tau_{i-h}^{F} \psi_{i}^{F} s x_{i}^{0 F}$ and $\lim _{t \rightarrow+\infty} \frac{0}{\frac{d i s}{P_{i}^{M}}(h, t)} \lambda_{0}^{t}=\tau_{i-h}^{M} \psi_{i}^{M} s x_{i}^{0 M}$.

Assuming that the disability retirement rate is for females $\operatorname{dis}_{i} \sigma_{i}^{F}(h)$ and for males ${ }_{d i s} \sigma_{i}^{M}(h)$ and that if it is applied to actives it is possible to express retirees, as before with direct pensions:

$$
\begin{aligned}
& \operatorname{dis}_{i} P_{i}^{F}(h, t)={ }_{d i s} \sigma_{i-1}^{F}(h) l_{i-1}^{F}(h, t-1) \psi_{i-1}^{F}, \\
& \operatorname{dis}_{i} P_{i}^{M}(h, t)={ }_{d i s} \sigma_{i-1}^{M}(h) l_{i-1}^{M}(h, t-1) \psi_{i-1}^{M} .
\end{aligned}
$$


New disable retirees of age $i$ are proportional to $i$-th component of population vector. Dividing by the eigenvalue and passing to the limit, for females and males, it is respectively:

$$
\begin{aligned}
& \lim _{t \rightarrow+\infty} \frac{\operatorname{dis}_{i s} P_{i}^{F}(h, t)}{\lambda_{0}^{t}}={ }_{d i s} \bar{\tau}_{i, h}^{F} s x_{i}^{0 F} ; \\
& \lim _{t \rightarrow+\infty} \frac{d i s P_{i}^{M}(h, t)}{\lambda_{0}^{t}}={ }_{d i s} \bar{\tau}_{i, h}^{M} s x_{i}^{0 M},
\end{aligned}
$$

where ${ }_{d i s} \bar{\tau}_{i, h}^{F}={ }_{d i s} \sigma_{i}^{F} \tau_{i, h}^{F},{ }_{d i s} \bar{\tau}_{i, h}^{M}={ }_{d i s} \sigma_{i}^{M} \tau_{i, h}^{M}$.

Considering that $v$ years ago, new invalidity retirees were active, we have $\lim _{t \rightarrow+\infty} \frac{d i s P_{i-v}^{F}(h, t-v)}{\lambda_{0}^{t}}=\frac{1}{\lambda_{0}^{v}} \lim _{t \rightarrow+\infty} \frac{d i s P_{i-v}^{F}(h, t-v)}{\lambda_{0}^{t-v}}=\frac{1}{\lambda_{0}^{v}} d i s \bar{\tau}_{i, h}^{F} s x_{i}^{0 F}$ and $\lim _{t \rightarrow+\infty} \frac{d i s P_{i-v}^{M}(h, t-v)}{\lambda_{0}^{t}}=\frac{1}{\lambda_{0}^{v}} \lim _{t \rightarrow+\infty} \frac{d i s P_{i-v}^{M}(h, t-v)}{\lambda_{0}^{t-v}}=\frac{1}{\lambda_{0}^{v}} d i s \bar{\tau}_{i, h}^{M} s x_{i}^{0 M}$

Passing to the limit of $\frac{\pi_{d i s}(t)}{T(t)}$ for disable pensions, using (6) and (7), doing the same considerations on the transformation coefficient and indicating with ${ }_{d i s} \omega_{j}^{F}$ and ${ }_{d i s} \omega_{j}^{M}$ the odds of surviving of a disable female and male, as for direct pensions, we have:

$$
\begin{aligned}
& \lim _{t \rightarrow \infty} \frac{\pi_{d i s}(t)}{T(t)}=\left(\frac{\pi_{d i s}}{T}\right)_{\infty}= \\
= & c_{0} \frac{\sum_{i=36}^{a-1} \sum_{v=0}^{i-61} \sum_{h=10}^{i-26}\left(S_{c}+B\left(1-\frac{h}{H}\right)\right) \cdot\left(\left(1+r^{\prime}\right)^{v} \sum_{0<J \leq h} d_{i-v-J, t-j}(1+r)^{-1}\right) \cdot D^{d i s}(i, v, h)}{\sum_{i, h}\left(d i s^{\bar{\tau}} \bar{\tau}_{i, h} s x_{i}^{0 F}+d i s^{\bar{\tau}} \bar{\tau}_{i, h} s x_{i}^{0 M}\right) d_{i, h}}
\end{aligned}
$$

where

$$
D^{d i s}(i, v, h)=\left(\frac{1}{\lambda_{0}^{v}} d i s \bar{\tau}_{i, h}^{F} s x_{i}^{0 F} \prod_{j=i-v}^{i-1} d i s \omega_{j}^{F}+\frac{1}{\lambda_{0}^{v}} d i s \bar{\tau}_{i, h}^{M} s x_{i}^{0 M} \prod_{j=i-v}^{i-1} d i s \omega_{j}^{M}\right)
$$

\subsubsection{For inability pensions}

In case of inability for accountants of age $i$, with $26<i<a$ and $5 \leq h \leq i-26$, indicating with ${ }^{i n a} \psi_{i}^{F}$ and ${ }^{i n a} \psi_{i}^{M}$ the inability probability for females and males, explaining depending on actives, we have:

$$
{ }_{i n a}^{0} P_{i+1}^{F}(h, t+1)=l_{i}^{F}(h, t) \omega_{i}^{F i n a} \psi_{i}^{F},{ }_{i n a}^{0} P_{i+1}^{M}(h, t+1)=l_{i}^{M}(h, t) \omega_{i}^{M i n a} \psi_{i}^{M} .
$$

For $t$ sufficiently large, by (2) and (3) we obtain:

$$
\begin{aligned}
& \quad{ }_{\text {ina }}^{0} P_{i}^{F}(h, t)=l_{i}^{F}(h, t) \omega_{i}^{F i n a} \psi_{i}^{F}=\tau_{i-h}^{F}{ }^{i n a} \psi_{i}^{F} x_{i}^{F}(t) \text { and } \\
& { }_{\text {ina }} P_{i}^{M}(h, t)=l_{i}^{M}(h, t) \omega_{i}^{M i n a} \psi_{i}^{M}=\tau_{i-h}^{M} \text { ina } \psi_{i}^{M} x_{i}^{M}(t) .
\end{aligned}
$$

Applying the Theorem 1 for females and males, we have:

$$
\lim _{t \rightarrow+\infty} \frac{{ }_{i n a} P_{i}^{F}(h, t)}{\lambda_{0}^{t}}=\tau_{i-h}^{F}{ }^{i n a} \psi_{i}^{F} s x_{i}^{0 F} \text { and } \lim _{t \rightarrow+\infty} \frac{{ }_{i n a} P_{i}^{M}(h, t)}{\lambda_{0}^{t}}=\tau_{i-h}^{M}{ }^{i n a} \psi_{i}^{M} s x_{i}^{0 M} \text {. }
$$


Supposing that the inability retirement rate for females and males are ${ }_{i n a} \sigma_{i}^{F}(h)$ and ${ }_{i n a} \sigma_{i}^{M}(h)$. Appling these rates to actives it is possible to express the inability retirees, in the same way as for the directs:

$$
\begin{gathered}
{ }_{i n a} P_{i}^{F}(h, t)={ }_{i n a} \sigma_{i-1}^{F}(h) l_{i-1}^{F}(h, t-1) \psi_{i-1}^{F}, \\
{ }_{i n a} P_{i}^{M}(h, t)={ }_{i n a} \sigma_{i-1}^{M}(h) l_{i-1}^{M}(h, t-1) \psi_{i-1}^{M} .
\end{gathered}
$$

The new inability retirees of age $i$ are proportional to the $i$-th component of the population vector. Dividing by the $t$-th power of the eigenvalue and passing to the limit, it is possible to obtain, for females and males respectively:

$$
\begin{aligned}
& \lim _{t \rightarrow+\infty} \frac{{ }_{i n a} P_{i}^{F}(h, t)}{\lambda_{0}^{t}}={ }_{i n a} \bar{\tau}_{i, h}^{F} s x_{i}^{0 F} ; \\
& \lim _{t \rightarrow+\infty} \frac{{ }_{i n a} P_{i}^{M}(h, t)}{\lambda_{0}^{t}}={ }_{i n a} \bar{\tau}_{i, h}^{M} s x_{i}^{0 M},
\end{aligned}
$$

where ${ }_{i n a} \bar{\tau}_{i, h}^{F}={ }_{i n a} \sigma_{i}^{F} \tau_{i, h}^{F},{ }_{i n a} \bar{\tau}_{i, h}^{M}={ }_{i n a} \sigma_{i}^{M} \tau_{i, h}^{M}$.

Considering that $v$ years ago, the new retirees with disability were active, it is possible to write the limit in this way:

$$
\begin{aligned}
& \lim _{t \rightarrow+\infty} \frac{i n a P_{i-v}^{F}(h, t-v)}{\lambda_{0}^{t}}=\frac{1}{\lambda_{0}^{v}} \lim _{t \rightarrow+\infty} \frac{i n a P_{i-v}^{F}(h, t-v)}{\lambda_{0}^{t-v}}=\frac{1}{\lambda_{0}^{v}} i n a \bar{\tau}_{i, h}^{F} s x_{i}^{0 F} \text { and } \\
& \lim _{t \rightarrow+\infty} \frac{i n a P_{i-v}^{M}(h, t-v)}{\lambda_{0}^{t}}=\frac{1}{\lambda_{0}^{v}} \lim _{t \rightarrow+\infty} \frac{i n a P_{i-v}^{M}(h, t-v)}{\lambda_{0}^{t-v}}=\frac{1}{\lambda_{0}^{v}}{ }_{i n a} \bar{\tau}_{i, h}^{M} s x_{i}^{0 M} .
\end{aligned}
$$

Passing to the limit of $\frac{\pi_{i n a}(t)}{T(t)}$ for inability pensions, using 8 and $\sqrt{9}$, doing the same considerations on transformation coefficient and denoted by ina $\omega_{j}^{F}$ and ${ }_{\text {ina }} \omega_{j}^{M}$ the survival probability of a female and a male with disability, as for direct pension, it is possible to write:

$$
\begin{aligned}
& \lim _{t \rightarrow \infty} \frac{\pi_{i n a}(t)}{T(t)}=\left(\frac{\pi_{i n a}}{T}\right)_{\infty}= \\
= & c_{0} \frac{\sum_{i=31}^{a-1} \sum_{v=0}^{i-61} \sum_{h=5}^{i-26}\left(S_{c}+B\left(1-\frac{h}{H}\right)\right) \cdot\left(\left(1+r^{\prime}\right)^{v} \sum_{0<J \leq h} d_{i-v-J, t-j}(1+r)^{-1}\right) \cdot D^{i n a}(i, v, h)}{\sum_{i, h}\left({ }_{\text {ina }} \bar{\tau}_{i, h}^{F} s x_{i}^{0 F}+{ }_{i n a} \bar{\tau}_{i, h}^{M} s x_{i}^{0 M}\right) d_{i, h}}
\end{aligned}
$$

where

$$
D^{i n a}(i, v, h)=\left(\frac{1}{\lambda_{0}^{v} i n a} \bar{\tau}_{i, h}^{F} s x_{i}^{0 F} \prod_{j=i-v}^{i-1} i n a \omega_{j}^{F}+\frac{1}{\lambda_{0}^{v} i n a} \bar{\tau}_{i, h}^{M} s x_{i}^{0 M} \prod_{j=i-v}^{i-1} i n a \omega_{j}^{M}\right)
$$

\subsubsection{For indirect pensions}

It is necessary to remember that the model takes into account only the widows and the widowers, after 5 years of contribution. It is possible to express the number of indirect retirees starting from the actives, as follows:

$$
{ }_{i n d}^{0} P_{k}^{F}(h, t)=\sum_{i=31}^{a-1} l_{i}^{M}(h, t-1)\left(1-\omega_{i}^{M}\right)_{k}^{w i d} \theta_{i}^{M}=\sum_{i=31}^{a-1} \tau_{i-h k}^{M}{ }^{w i d} \theta_{i}^{M} x_{i}^{M}(t),
$$


${ }_{i n d}^{0} P_{k}^{M}(h, t)=\sum_{i=31}^{a-1} l_{i}^{F}(h, t-1)\left(1-\omega_{i}^{F}\right)_{k}^{w i d} \theta_{i}^{F}=\sum_{i=31}^{a-1} \tau_{i-h k}^{F} \underset{\text { wid }}{\theta_{i}^{F}} x_{i}^{F}(t)$ where $i$ is assignor's age and $k$ is widow(er)'s age who receives the indirect pension.

If $h<5$ it is not considered in the model due to the fact it is out of the dynamic:

$$
\begin{aligned}
& { }_{i n d}^{v} R C_{i}^{M}(h, t)=\sum_{i=26}^{a-1} l_{i}^{F}(h, t-1)\left(1-\omega_{i}^{F}\right)_{k}^{w i d} \theta_{i}^{F}=\sum_{i=26}^{a-1} \tau_{i-h k}^{F} \underset{w i d}{\theta_{i}^{F}} x_{i}^{F}(t) \\
& { }_{i n d}^{v} R C_{i}^{F}(h, t)=\sum_{i=26}^{a-1} l_{i}^{M}(h, t-1)\left(1-\omega_{i}^{M}\right)_{k}^{w i d} \theta_{i}^{M}=\sum_{i=26}^{a-1} \tau_{i-h k}^{M} \underset{w i d}{\theta_{i}^{M}} x_{i}^{M}(t) .
\end{aligned}
$$

Applying the Theorem 1 for females and males:

$\lim _{t \rightarrow+\infty} \frac{{ }_{i n d} P_{k}^{F}(h, t)}{\lambda_{0}^{t}}=\tau_{i-h}^{F}{ }^{i n d} \psi_{i}^{F} s x_{i}^{0 F}$ and $\lim _{t \rightarrow+\infty} \frac{{ }^{0}{ }_{i n d} P_{k}^{M}(h, t)}{\lambda_{0}^{t}}=\tau_{i-h}^{M}{ }^{i n d} \psi_{i}^{M} s x_{i}^{0 M}$.

Assuming that ${ }_{i n d} \sigma_{i}^{F}(h)$ and ${ }_{i n d} \sigma_{i}^{M}(h)$ are the females and males indirect retirement rate to apply to the actives, considering the odds of a male active, who dies, leaves a widow and a female active, who dies, leaves a widower:

$$
\begin{aligned}
& { }_{i n d} P_{i}^{F}(h, t)={ }_{i n d} \sigma_{i-1}^{F}(h) l_{i-1}^{F}(h, t-1) \psi_{i-1}^{F}, \\
& { }_{i n d} P_{i}^{M}(h, t)={ }_{i n d} \sigma_{i-1}^{M}(h) l_{i-1}^{M}(h, t-1) \psi_{i-1}^{M} .
\end{aligned}
$$

Dividing by the $t$-th component of the eigenvalue and passing to the limit, it is possible to obtain for females and males, respectively:

$$
\begin{aligned}
& \lim _{t \rightarrow+\infty} \frac{{ }_{i n d} P_{k}^{F}(h, t)}{\lambda_{0}^{t}}={ }_{i n d} \bar{\tau}_{i, h}^{F} s x_{i}^{0 F} \\
& \lim _{t \rightarrow+\infty} \frac{i n d P_{k}^{M}(h, t)}{\lambda_{0}^{t}}={ }_{i n d} \bar{\tau}_{i, h}^{M} s x_{i}^{0 M},
\end{aligned}
$$

where ${ }_{i n d} \bar{\tau}_{i, h}^{F}={ }_{i n d} \sigma_{i}^{F} \tau_{i, h}^{F},{ }_{i n d} \bar{\tau}_{i, h}^{M}={ }_{i n d} \sigma_{i}^{M} \tau_{i, h}^{M}$.

Considering that $v$ years ago the indirect retirees were spouses of an active accountant, the limits are:

$$
\begin{aligned}
& \lim _{t \rightarrow+\infty} \frac{i n d P_{k-v}^{F}(h, t-v)}{\lambda_{0}^{t}}=\frac{1}{\lambda_{0}^{v}} \lim _{t \rightarrow+\infty} \frac{i n d P_{k-v}^{F}(h, t-v)}{\lambda_{0}^{t-v}}=\frac{1}{\lambda_{0}^{v}}{ }_{i n d} \bar{\tau}_{i, h}^{F} s x_{i}^{0 F} \\
& \lim _{t \rightarrow+\infty} \frac{i n d P_{k-v}^{M}(h, t-v)}{\lambda_{0}^{t}}=\frac{1}{\lambda_{0}^{v}} \lim _{t \rightarrow+\infty} \frac{i n d P_{k-v}^{M}(h, t-v)}{\lambda_{0}^{t-v}}=\frac{1}{\lambda_{0}^{v}} i n d_{i, h} \bar{\tau}_{i, h}^{M} s x_{i}^{0 M} .
\end{aligned}
$$

Passing to the limit of $\frac{\pi_{i n d}(t)}{T(t)}$ for indirect pensions, by 10 and $\sqrt{11}$, with the same considerations on the transformation coefficient and indicating with ${ }_{i n d} \omega_{j}^{F}$ and ${ }_{i n d} \omega_{j}^{M}$ the survival probability of a female and a male inderect retiree the limit is:

$$
\begin{aligned}
& \lim _{t \rightarrow \infty} \frac{\pi_{i n d}(t)}{T(t)}=\left(\frac{\pi_{i n d}}{T}\right)_{\infty}= \\
& =c_{0} \frac{\sum_{i=31}^{a-1} \sum_{v=0}^{i-61} \sum_{h=5}^{i-26}\left(\alpha+B\left(1-\frac{h}{H}\right)\right) \cdot\left(\left(1+r^{\prime}\right)^{v} \sum_{0<J \leq h} d_{i-v-J, t-j}(1+r)^{-1}\right) \cdot D^{i n d}(i, v, h)}{\sum_{i, h}\left(i n d^{\bar{\tau}_{i, h}} \bar{T}_{i}^{0}{ }_{i} F+{ }_{i n d} \bar{\tau}_{i, h}^{M} s x_{i}^{0 M}\right) d_{i, h}}
\end{aligned}
$$


where

$$
D^{i n d}(i, v, h)=\left(\frac{1}{\lambda_{0}^{v}} i n d \bar{\tau}_{i, h}^{F} s x_{i}^{0 F} \prod_{j=i-v}^{i-1} i n d \omega_{j}^{F}+\frac{1}{\lambda_{0}^{v}}{ }_{i n d} \bar{\tau}_{i, h}^{M} s x_{i}^{0 M} \prod_{j=i-v}^{i-1} i n d \omega_{j}^{M}\right)
$$

2.4.5 For reversibility pensions To determine the limit of the ratio between reversibility pensions and income is necessary to make explicit widows and widowers in function of active workers.

Indicating with $v$ the retirement age of widows and widowers of age $k$ (assuming that marriage is not contracted before 18 years old, $\beta \geq 18$ ) and with $i$ the assignor age, the reversibility in relation with direct is:

$$
\begin{aligned}
& { }_{r e v}^{0} P_{k}^{F}(h, t)=\sum_{i=\beta}^{104} \sum_{v=0}^{104-\beta} \underset{d i r}{v} P_{i}^{M}(h, t-1)\left(1-\omega_{i}^{M}\right)_{k}^{w i d} \theta_{i}^{M}= \\
& =\sum_{i=\beta}^{104} \sum_{v=0}^{104-\beta}\left(l_{i-v-1}^{F}(h, t-v-1) \omega_{i-v-1}^{F}-l_{i-v}^{F}(h+1, t-v)\right)\left(1-\omega_{i}^{M}\right)_{k}^{w i d} \theta_{i}^{M} ; \\
& { }_{r e v}^{0} P_{k}^{M}(h, t)=\sum_{i=\beta}^{104} \sum_{v=0}^{104-\beta} \underset{d i r}{v} P_{i}^{F}(h, t-1)\left(1-\omega_{i}^{F}\right)_{k}^{w i d} \theta_{i}^{F}= \\
& =\sum_{i=\beta}^{104} \sum_{v=0}^{104-\beta}\left(l_{i-v-1}^{M}(h, t-v-1) \omega_{i-v-1}^{M}-l_{i-v}^{M}(h+1, t-v)\right)\left(1-\omega_{i}^{F}\right)_{k}^{w i d} \theta_{i}^{F} .
\end{aligned}
$$

Applying the Theorem 1 for females and males, the limits are:

$$
\lim _{t \rightarrow+\infty} \frac{{ }_{r e v} P_{k}^{F}(h, t)}{\lambda_{0}^{t}}=\tau_{i-h}^{F}{ }^{r e v} \psi_{i}^{F} s x_{i}^{0 F} \text { and } \lim _{t \rightarrow+\infty} \frac{{ }_{r e v}^{0} P_{k}^{M}(h, t)}{\lambda_{0}^{t}}=\tau_{i-h}^{M}{ }^{r e v} \psi_{i}^{M} s x_{i}^{0 M} \text {. }
$$

Assuming that the reversibility retirement rate is constant over time and equal to ${ }_{r e v} \sigma_{i}^{F}(h)$ and ${ }_{r e v} \sigma_{i}^{M}(h)$ for gender, and considering the odds that a male retiree who dies leaves a widow and that a female who dies leaves a widower, it is possible to write:

$$
\begin{aligned}
& \operatorname{rev}_{i} P_{i}^{F}(h, t)={ }_{r e v} \sigma_{i-1}^{F}(h) l_{i-1}^{F}(h, t-1) \psi_{i-1}^{F}, \\
& \operatorname{rev}_{i}^{M}(h, t)={ }_{r e v} \sigma_{i-1}^{M}(h) l_{i-1}^{M}(h, t-1) \psi_{i-1}^{M} .
\end{aligned}
$$

Hence, the limits are respectively:

$$
\begin{aligned}
\lim _{t \rightarrow+\infty} \frac{r e v}{P_{k}^{F}(h, t)} & ={ }_{r e v} \bar{\tau}_{i, h}^{F} s x_{i}^{0 F} ; \\
\lim _{t \rightarrow+\infty} \frac{r e v}{P_{k}^{M}(h, t)} & ={ }_{r e v} \bar{\tau}_{i, h}^{M} s x_{i}^{0 M},
\end{aligned}
$$

where ${ }_{r e v} \bar{\tau}_{i, h}^{F}={ }_{r e v} \sigma_{i}^{F} \tau_{i, h}^{F}$, rev $_{i, h} \bar{\tau}_{i,{ }_{r e v}}^{M} \sigma_{i}^{M} \tau_{i, h}^{M}$. Considering that $v$ years ago, new survival retirees were spouses of a retired accountant, the limits are:

$$
\begin{aligned}
& \lim _{t \rightarrow+\infty} \frac{r e v}{P_{k-v}^{F}(h, t-v)} \lambda_{0}^{t}=\frac{1}{\lambda_{0}^{v}} \lim _{t \rightarrow+\infty} \frac{r e v P_{k-v}^{F}(h, t-v)}{\lambda_{0}^{t-v}}=\frac{1}{\lambda_{0}^{v}} r e v \bar{\tau}_{i, h}^{F} s x_{i}^{0 F} \\
& \lim _{t \rightarrow+\infty} \frac{r e v}{P_{k-v}^{M}(h, t-v)} \lambda_{0}^{t}=\frac{1}{\lambda_{0}^{v}} \lim _{t \rightarrow+\infty} \frac{r e v P_{k-v}^{M}(h, t-v)}{\lambda_{0}^{t-v}}=\frac{1}{\lambda_{0}^{v}} r e v \bar{\tau}_{i, h}^{M} s x_{i}^{0 M} .
\end{aligned}
$$

Passing to the limit of $\frac{\pi_{r e v}(t)}{T(t)}$ for survival pensions, using $\sqrt{12}$ and $\sqrt{13}$, making the same considerations on the transformation coefficient and indicating with 
${ }_{r e v} \omega_{j}^{F}$ and ${ }_{r e v} \omega_{j}^{M}$ the survival probability of a female and male retiree, respectively, the limit is:

$$
\begin{aligned}
& \lim _{t \rightarrow \infty} \frac{\pi_{r e v}(t)}{T(t)}=\left(\frac{\pi_{r e v}}{T}\right)_{\infty}= \\
= & c_{0} \frac{\sum_{i=31}^{a-1} \sum_{v=0}^{i-61} \sum_{h=20}^{i-26}\left(S_{c}+B\left(1-\frac{h}{H}\right)\right) \cdot\left(\left(1+r^{\prime}\right)^{v} \sum_{0<J \leq h} d_{i-v-J, t-j}(1+r)^{-1}\right) \cdot D^{r e v}(i, v, h)}{\sum_{i, h}\left(\operatorname{rev}_{i, h} \bar{\tau}_{i, h}^{F} s x_{i}^{0 F}+\operatorname{rev}_{i, h}^{M} s x_{i}^{0 M}\right) d_{i, h}}
\end{aligned}
$$

where

$$
D^{r e v}(i, v, h)=\left(\frac{1}{\lambda_{0}^{v}} r e v \bar{\tau}_{i, h}^{F} s x_{i}^{0 F} \prod_{j=i-v}^{i-1} r e v \omega_{j}^{F}+\frac{1}{\lambda_{0}^{v} r e v} \bar{\tau}_{i, h}^{M} s x_{i}^{0 M} \prod_{j=i-v}^{i-1} r e v \omega_{j}^{M}\right)
$$

Now it is necessary to formalize the limit of the ratio between fixed pension and compensation. The previously calculated limits of retirees, of all categories, with respect to the active population, according to Theorem 1, are indicated with a formal simplified notation, as follows 46

$$
\begin{aligned}
& \operatorname{LimDir}=\left[\frac{1}{\lambda_{0}^{v}} \bar{\tau}_{i, h}^{F} s x_{i}^{0 F} \prod_{j=i-v}^{i-1} \omega_{j}^{F}+\frac{1}{\lambda_{0}^{v}} \bar{\tau}_{i, h}^{M} s x_{i}^{0 M} \prod_{j=i-v}^{i-1} \omega_{j}^{M}\right] \\
& \operatorname{LimInv}=\left[\frac{1}{\lambda_{0}^{v} i n v} \bar{\tau}_{i, h}^{F} s x_{i}^{0 F} \prod_{j=i-v}^{i-1} i n v \omega_{j}^{F}+\frac{1}{\lambda_{0}^{v} i n v} \bar{\tau}_{i, h}^{M} s x_{i}^{0 M} \prod_{j=i-v}^{i-1}{ }^{i n v} \omega_{j}^{M}\right] \\
& \text { LimIna }=\left[\frac{1}{\lambda_{0}^{v} i n a} \bar{\tau}_{i, h}^{F} s x_{i}^{0 F} \prod_{j=i-v}^{i-1} i n a \omega_{j}^{F}+\frac{1}{\lambda_{0}^{v} i n a} \bar{\tau}_{i, h}^{M} s x_{i}^{0 M} \prod_{j=i-v}^{i-1} i n a \omega_{j}^{M}\right] \\
& \text { LimInd }=\left[\frac{1}{\lambda_{0}^{v} i n d} \bar{\tau}_{i, h}^{F} s x_{i}^{0 F} \prod_{j=i-v}^{i-1} i n d \omega_{j}^{F}+\frac{1}{\lambda_{0}^{v}} i n d \bar{\tau}_{i, h}^{M} s x_{i}^{0 M} \prod_{j=i-v}^{i-1}{ }^{i n d} \omega_{j}^{M}\right]+ \\
& +\left[\frac{1}{\lambda_{0}^{v}}{ }_{i n d} R C_{i-v}^{F}(h, \Omega-1-v)+\frac{1}{\lambda_{0}^{v}}{ }_{i n d} R C_{i-v}^{M}(h, \Omega-1-v)\right] \\
& \text { LimRev }=\left[\frac{1}{\lambda_{0}^{v}} \operatorname{rev} \bar{\tau}_{i, h}^{F} s x_{i}^{0 F} \prod_{j=i-v}^{i-1}{ }^{i d} \omega_{j}^{F}+\frac{1}{\lambda_{0}^{v}} \operatorname{rev} \bar{\tau}_{i, h}^{M} s x_{i}^{0 M} \prod_{j=i-v}^{i-1}{ }^{i d} \omega_{j}^{M}\right] .
\end{aligned}
$$

Therefore, the sought limit is:

$$
\begin{aligned}
\lim _{t \rightarrow \infty} \frac{\pi_{f i x}(t)}{T(t)} & =\lim _{t \rightarrow \infty} \frac{F(t) \sum_{i=70}^{\Omega-1} \sum_{v=0}^{44}\left({ }^{v} P_{i}^{F}(t)+{ }^{v} P_{i}^{M}(t)\right)}{\sum_{i, h}\left(\bar{\tau}_{i, h}^{F} s x_{i}^{0 F}+\bar{\tau}_{i, h}^{M} s x_{i}^{0 M}\right) d_{i h}}= \\
& =\frac{F(t)(\text { LimDir }+ \text { LimInv }+ \text { LimIna }+ \text { LimInd }+ \text { LimRev })}{\sum_{i, h}\left(\bar{\tau}_{i, h}^{F} s x_{i}^{0 F}+\bar{\tau}_{i, h}^{M} s x_{i}^{0 M}\right) d_{i h}}
\end{aligned}
$$

${ }^{46}$ Cfr. [4]. 
AN ACTUARIAL MATHEMATICAL MODEL FOR A NEW PENSION PHILOSOPHY... 51

Now it is possible to proceed with the formalization of the PAYG contribution rate of the tendential equilibrium considering all the possible pension components, both for the contribution share and for the fixed one which expresses the percentage cost in terms of annual income.

$\frac{1}{\phi}\left(\frac{\pi}{T}\right)_{\infty}=\left(\frac{\pi_{d i r}}{T}\right)_{\infty}+\left(\frac{\pi_{i n v}}{T}\right)_{\infty}+\left(\frac{\pi_{i n a}}{T}\right)_{\infty}+\left(\frac{\pi_{i n d}}{T}\right)_{\infty}+\left(\frac{\pi_{r e v}}{T}\right)_{\infty}+\left(\frac{\pi_{f i x}}{T \phi}\right)_{\infty}$.

Assuming $G=\frac{1}{\phi}\left(\frac{\pi}{T}\right)_{\infty}$ it is possible to rewrite equation (1) that becomes, with a substitution, $G=\frac{1-\phi}{\phi} A$, where:

- $A$ is the PAYG contribution rate of tendential equilibrium that can be called average tendential substitution rate in hypothesis of demographic equilibrium;

- $\phi$ is the contribution rate provided for CNPADC that is equal for all members but can be revised in every moment;

- The pension shares are two, the DC financed by active members and the fixed one financed by an additional contribution from both retirees and active chartered accountant or, alternatively, by drawing on the supplementary contribution.

If, as required by law, the portion of ratio of direct pensions (even though on average) must generate a substitution rate (of a tendential equilibrium) at least of $60 \%$, which obviously carries the average reversibility, it is clear that in this logic, all pension structure is subjected to a change. So we have a PAYG contribution rate of the tendential equilibrium with an intrinsic clause of social guarantee of adequacy on a collective basis and therefore systemic.

\section{Applications and results}

The elaboration of data available in the actuarial balance was made using the model built above, with the aim to offer a contribution to the reflection on the pension adequacy issue with respect to a PAYG contribution rate of tendential equilibrium.

In order to test the hypotheses of the law proposal, it has been necessary to determine technical demographic, financial and economical basis referring to the various types of pensions of CNPADC (direct, indirect, reversibility, disability, inability) and to the amount of the consequent disbursements that CNPADC will face in the future. For the application of the actuarial mathematical model, adapted to the rules in force in CNPADC, MatLah 47 was used.

Figures with results of the application are reported in the appendix.

In the search for a contribution rate of equilibrium that allows to reach the replacement rate that law proposal sets as target, as shown below in the table

\footnotetext{
47 Cfr. [18].
} 
2 , it can be stated that the gross replacement rate of at least $60 \%$ is reached with a rate of around $15,5 \%$ considering the defined contribution and the fixed rate.

\begin{tabular}{|c|c|}
\hline Contribution rate & Substitution rate \\
\hline $12 \%$ & $34,52 \%$ \\
\hline $13 \%$ & $41,50 \%$ \\
\hline $14 \%$ & $49,22 \%$ \\
\hline $15 \%$ & $57,69 \%$ \\
\hline $16 \%$ & $66,96 \%$ \\
\hline
\end{tabular}

Table 2 - Substitution rate per contribution rate

The CNPADC substitution rate is $33,2 \%$ with a contribution rate of $12 \%$, as it is possible to see in the actuarial balance.

The disability, inability, indirect and reversibility pensions are not so important to the weight of the substitution rate of tendential equilibrium, while the direct pension is the predominant component to determine almost the entire substitution rate.

\section{Conclusions}

The actuarial mathematical formalization of the law proposal is on a collective and not an individual basis referring to Theorem 1 and therefore to the asymptotic demographic equilibrium of the entire population. In this paper, the model has been adapted to CNPADC members and its rules even though the logic can be adapted to any pension funds. Based on this formalization, it is highlighted that the variables involved to guarantee an average tendency substitution rate of at least $60 \%$, minimal percentage to guarantee a fair lifestyle on direct pensions are: retirement age, seniority in contributions and rates.

Considering that, to maintain the principle of adequacy indicated by Cazzola and Treu and repeatedly requested by the European Union, it is necessary to increase the variables, as already provided for by the latest Monti-Fornero reform law, present in the Save Italy Decree (from "quota 100" of the Conte government, members of social security funds are excluded).

The rigor of the here proposed model allows to formulate indications on how to involve some parameters, with the limitation of the collective logic. The trend equilibrium rate between the defined contribution pension and the fixed one, came to light from the processing through MatLab, is around 15,5\%, supposing a retirement age of 70 years. Hence, the replacement rate of the direct pension, that the law proposal provides for at least equal to $60 \%$, needs to be increased from $12 \%$ currently due from members of CNPADC. It should be highlighted that, hypothesizing a postponement of the retirement age to 73 years, the tendential equilibrium rate would not be favourably impacted, but 
instead, it would lead to a penalty of a few tenths of a percentage (compared to a rate of $16 \%$, the replacement rate would be $66,04 \%$ compared to $66,96 \%$ with a retirement age of 70 years). This is due to the fact that from the age of 65 , the incomes of registered accountant tend to decrease. In addition, postponing the retirement age, CNPADC members will receive pension for less years and so the deposited contributions amount will be rubbed on less years.

The law proposal n. 3035, referring to the basic public pension, provides for a partition of contribution rate between employee and employer which is obviously inapplicable for professionals and, specifically, for accountants. It can be considered that if the two thirds, on average equal to about 17,33 , paid by employers were deposited by professionals, the latter would have a tendential replacement rate, based on this equilibrium trend rate, equal to about $77,03 \%$.

\section{Appendix}

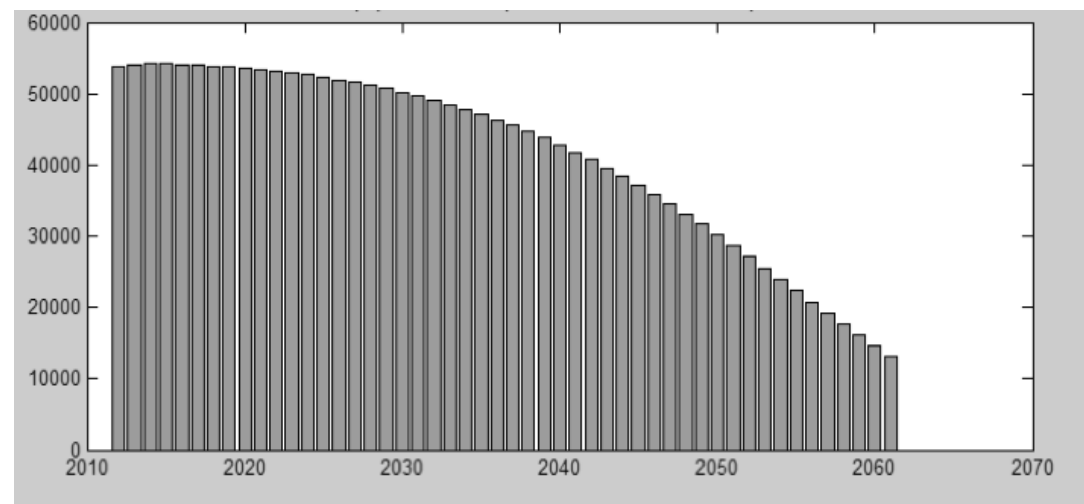

Figure 1: Projection of the state vector of the population 


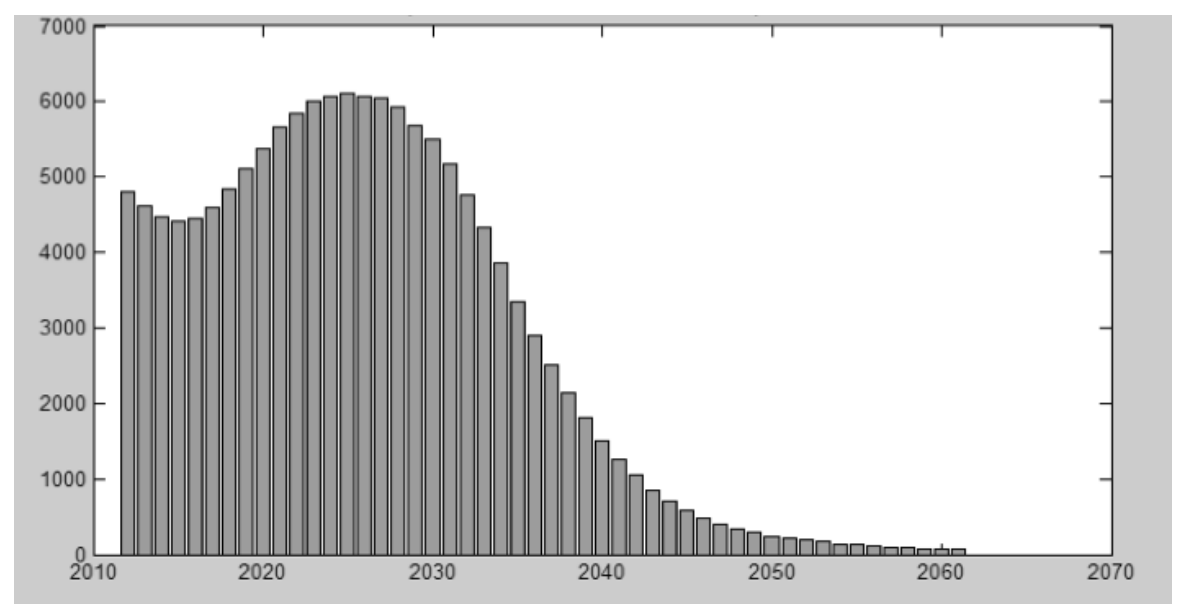

Figure 2: Projection of the total number of pensioners for all pension types

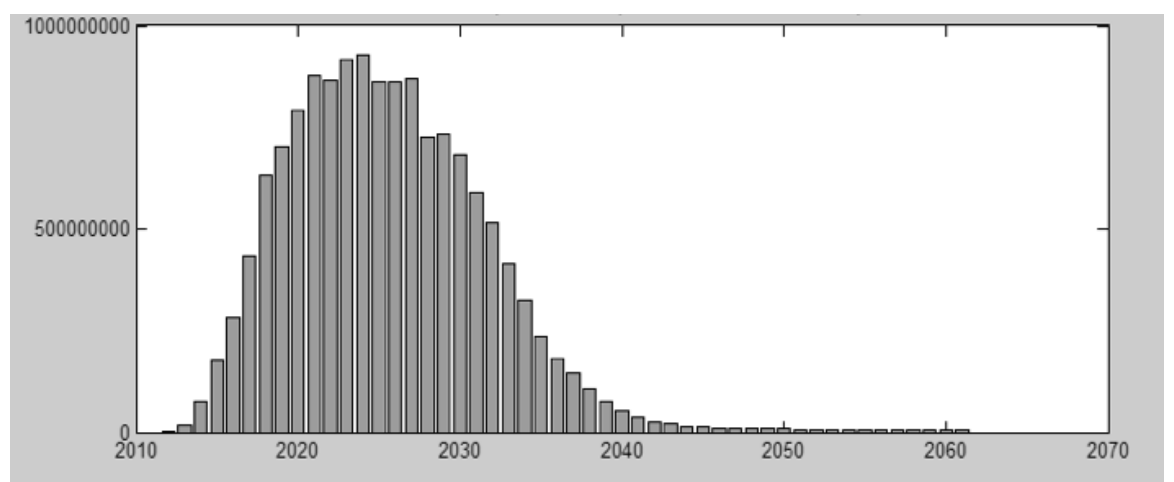

Figure 3: Projection of the amount of contribution benefits for all pension types

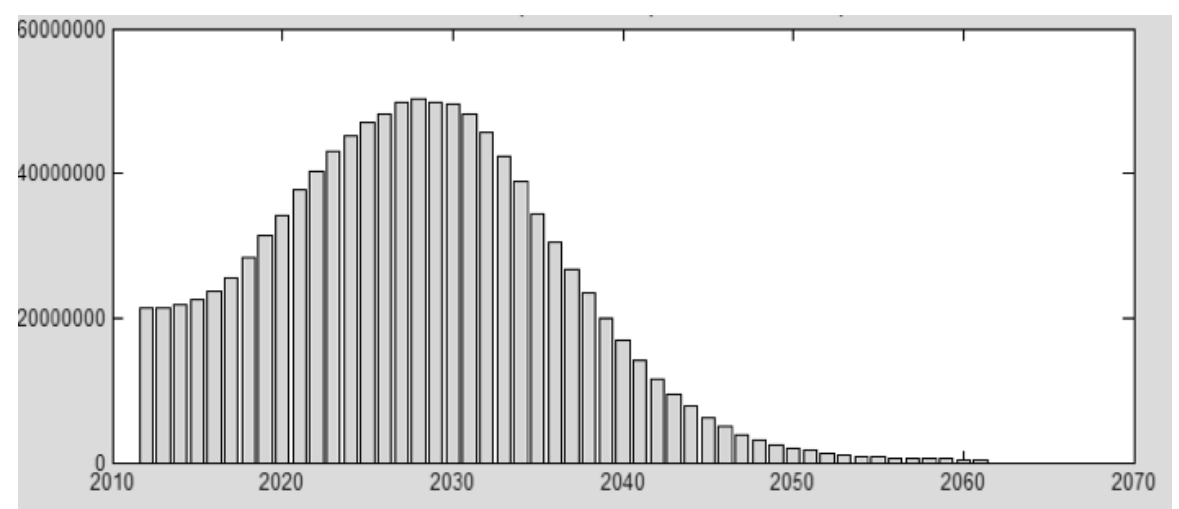

Figure 4: Projection of the amount of the fixed fee service according to the Cazzola-Treu logic 


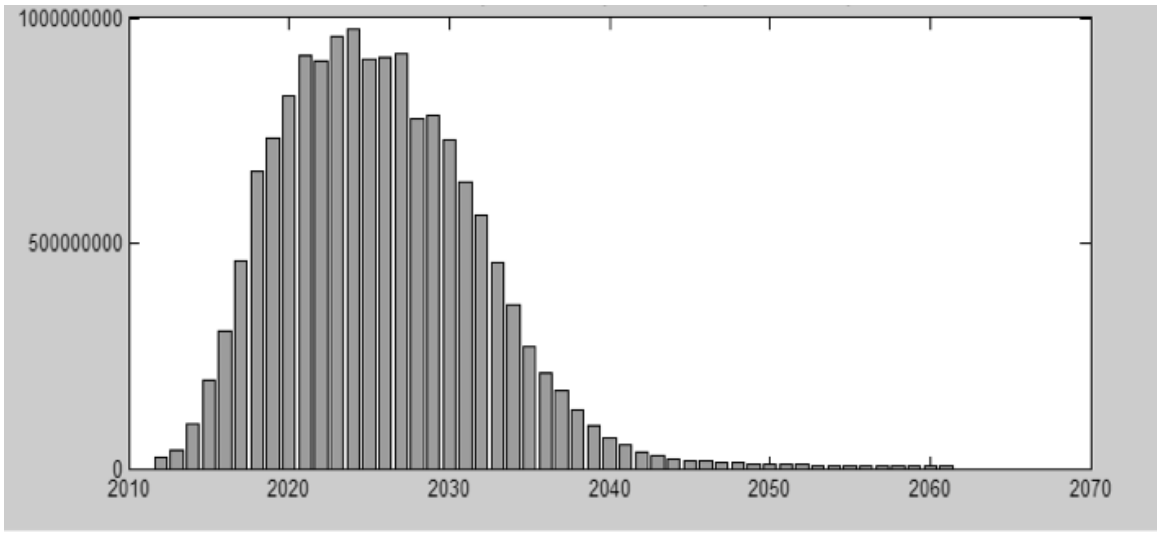

Figure 5: Projection of the total amount of pension benefits in a fixed contribution rate 


\section{References}

1. Angrisani M, Attias A (2004), A mathematical model for the management of an invalidity old age survivor pension fund: the Exact Individual Trajectories Method, PU.M.A. Pure Mathematics and Applications, Vol. 15, pp. 1-15.

2. Angrisani M, Attias A, Bianchi S, Varga Z (2006), Demographic dynamic for the pay-as-you-go pension system, PU.M.A. Pure Mathematics and Applications, Vol. 15, n. 4, pp. 357-374.

3. Annibali A (2017), CNPADC Actuarial Balance, projections: 2017 - 2066.

4. Attias A (2007), Demographic equilibrium and flexibility of the pension system, Proceedings XIII Conference on Actuarial Risk Theory, pp. 5-37, Aracne Edition.

5. Attias A (2012), A two-component public pension system under demographic equilibrium: a substitution rate for benefit adequacy, Proceedings XVIII Conference on Actuarial Risk Theory, pp. 37-74, Libellula Edition.

6. Attias A, Arezzo MF, Pianese A, Varga Z (2016), A comparison of two legislative approaches to the pay-as-you-go pension system in terms of adequacy. The Italian case, Insurance: Mathematics and Economics Vol. 68, pp. 203-211.

7. Berman A, Plemmons RJ (1994), Nonnegative matrices in mathematical sciences, Siam, Philadelphia.

8. Ciavalini S (2013), Adeguatezza delle prestazioni previdenziali: i futuri pensionati assaliranno il forno delle grucce? Il modello matematico attuariale della proposta Cazzola-Treu adattato e applicato alla realtà della $C N P A D C$, Tesi di laurea in Finanza e Assicurazioni.

9. Coppini MA (1989), The INPS model and the first projections to 2010, INPS, supplement to the magazine Previdenza Sociale, Rome, Italy.

10. European Commission (DG ECFIN) and Economic Policy Committee (AWG) (2009). Ageing report: Economic and budgetary projections for the EU-27 Member States (2008-2060). European Economy, nº 2.

11. European Commission (2010), Green Paper towards adequate, sustainable and safe European pension systems, Bruxelles.

12. European Commission (2018), Pension Adequacy Report 2018. Current and future income adequacy in old age in EU, vol. 2, Publications Office of the European Union, Luxembourg. 
13. Golany B, Thore, S (1997), The economic and social performances of Nations: efficiency and returns to scale, Socio-Economic Planning Sciences, Vol. 31, No. 3, pp. 191-204.

14. Leslie PH (1945), On the use of matrices in certain population mathematics, Biometrika, 33, n. 3, p. 183-212, Oxford University Press, UK.

15. Leslie PH (1948), Some further notes on the use of matrices in certain population mathematics, Biometrika, Vol. 35, n. 3-4, p. 213-245, Oxford University Press, UK.

16. Ministerial Commission for the evaluation of the effects of law n. 335/95 and subsequent provisions (2001), Verification of the social security system pursuant to law 335/95 and subsequent provisions, with a view to developing competitiveness and equity, Italy.

17. Ministry of Economy and Finance, RGS (2017), Country Fiche on public pensions for the Ageing Report 2018, EPC - WGA, Italy.

18. The Mathworks (2009), MATLAB - The language of technical computing, version 7.9.0, Natick MA. 